Mathematical Sciences And Applications E-Notes

Volume 3 No. 2 Pp. 1-19 (2015) @ MSAEN

\title{
SOME HERMITE-HADAMARD AND SIMPSON LIKE INEQUALITIES FOR $s$-GEOMETRICALLY CONVEX FUNCTION
}

\author{
MEVLÜT TUNÇ*, EBRU YÜKSEL** \\ (Communicated by Nihal YILMAZ ÖZGÜR)
}

\begin{abstract}
In the paper, the authors establish and generalize some new integral inequalities of Hermite-Hadamard and Simpson type for functions the power of the absolute of whose first derivative is $s$-geometrically convex.
\end{abstract}

\section{INTRODUCTION}

In this section, we will present definitions and some known results used in this paper.

Let $I$ be an interval in $\mathbb{R}$. Then $f: I \rightarrow \mathbb{R}, \emptyset \neq I \subseteq \mathbb{R}$ is said to be convex if

$$
f(t x+(1-t) y) \leq t f(x)+(1-t) f(y)
$$

for all $x, y \in I$ and $t \in[0,1]$.

Let $f: I \subseteq \mathbb{R} \rightarrow \mathbb{R}$ be a convex mapping defined on the interval $I$ of real numbers and $a, b \in I$, with $a<b$. The following double inequality:

$$
f\left(\frac{a+b}{2}\right) \leq \frac{1}{b-a} \int_{a}^{b} f(x) d x \leq \frac{f(a)+f(b)}{2}
$$

holds. This double inequality is known in the literature as the Hermite-Hadamard inequality for convex functions, see the papers and books [2]-[4], [6]-[10], [13]-[16].

The Simpson inequality proposes that the mapping $f:[a, b] \rightarrow \mathbb{R}$ is assumed to be four times continuously differentiable on the interval and $f^{(4)}$ to be bounded on $(a, b)$, that is,

$$
\left|\frac{1}{6}\left[f(a)+f(b)+4 f\left(\frac{a+b}{2}\right)\right]-\frac{1}{b-a} \int_{a}^{b} f(x) d x\right| \leq \frac{1}{1280}\left\|f^{(4)}\right\|_{\infty}(b-a)^{4},
$$

where $\left\|f^{(4)}\right\|_{\infty}=\sup _{t \in(a, b)}\left|f^{(4)}(t)\right|<\infty$. For some results which generalize, improve and extend the inequality (1.3), see the papers [1], [5], [11], [12].

Date: Received: January 6, 2015; Accepted: September 29, 2015.

2010 Mathematics Subject Classification. 26A15, 26A16, 26A33, 26A51, 26D10.

Key words and phrases. Convex function; Hermite-Hadamard integral inequality; Simpson integral inequality; s-geometrically convex function.

*Corresponding author. 
Definition 1.1. [8] Let $s \in(0,1]$. A function $f: I \subset \mathbb{R}_{0}=[0, \infty) \rightarrow \mathbb{R}_{0}$ is said to be s-convex in the second sense if

$$
f(t x+(1-t) y) \leq t^{s} f(x)+(1-t)^{s} f(y)
$$

for all $x, y \in I$ and $t \in[0,1]$.

It can be easily checked for $s=1, s$-convexity reduces to the ordinary convexity of functions defined on $[0, \infty)$.

Recently, in [15], the concept of geometrically and s-geometrically convex functions was introduced as follows.

Definition 1.2. [15] A function $f: I \subset \mathbb{R}_{+}=(0, \infty) \rightarrow \mathbb{R}_{+}$is said to be a geometrically convex function if

$$
f\left(x^{t} y^{1-t}\right) \leq[f(x)]^{t}[f(y)]^{1-t}
$$

for all $x, y \in I$ and $t \in[0,1]$.

Definition 1.3. [15] A function $f: I \subset \mathbb{R}_{+} \rightarrow \mathbb{R}_{+}$is said to be a $s$-geometrically convex function if

$$
f\left(x^{t} y^{1-t}\right) \leq[f(x)]^{t^{s}}[f(y)]^{(1-t)^{s}}
$$

for some $s \in(0,1]$, where $x, y \in I$ and $t \in[0,1]$.

If $s=1$ in (1.6), the $s$-geometrically convex function becomes a geometrically convex function on $\mathbb{R}_{+}$.

Example 1.1. [15] Let $f(x)=x^{s} / s, x \in(0,1], 0<s<1, q \geq 1$, and then the function

$$
\left|f^{\prime}(x)\right|^{q}=x^{(s-1) q}
$$

is monotonically decreasing on $(0,1]$. For $t \in[0,1]$, we have

$$
(s-1) q\left(t^{s}-t\right) \leq 0, \quad(s-1) q\left((1-t)^{s}-(1-t)\right) \leq 0 .
$$

Hence, $\left|f^{\prime}(x)\right|^{q}$ is $s$-geometrically convex on $(0,1]$ for $0<s<1$.

In recently [16], Zhang et al. proved some Hermite-Hadamard type inequalities for $s$-geometrically convex functions as followings:

Theorem 1.1. [16] Let $f: I \subset \mathbb{R}_{+} \rightarrow \mathbb{R}$ be a differentiable on $I^{\circ}$ such that $f^{\prime} \in L([a, b])$ for $0<a<b<\infty$. If $\left|f^{\prime}(x)\right|^{q}$ is s-geometrically convex and monotonically decreasing on $[a, b]$ for $q \geq 1$ and $s \in(0,1]$, then

$$
\left|f\left(\frac{a+b}{2}\right)-\frac{1}{b-a} \int_{a}^{b} f(x) d x\right| \leq \frac{b-a}{4}\left(\frac{1}{2}\right)^{1-1 / q} G_{1}\left(s, q ; g_{1}(\alpha), g_{2}(\alpha)\right)
$$

$$
\left|\frac{f(a)+f(b)}{2}-\frac{1}{b-a} \int_{a}^{b} f(x) d x\right| \leq \frac{b-a}{4}\left(\frac{1}{2}\right)^{1-1 / q} G_{1}\left(s, q ; g_{2}(\alpha), g_{1}(\alpha)\right)
$$

where

$$
g_{1}(\alpha)=\left\{\begin{array}{cl}
\frac{1}{2}, & \alpha=1, \\
\frac{\alpha \ln \alpha-\alpha+1}{[\ln \alpha]^{2}}, & \alpha \neq 1,
\end{array} \quad g_{2}(\alpha)=\left\{\begin{array}{cc}
\frac{1}{2}, & \alpha=1, \\
\frac{\alpha-\ln \alpha-1}{[\ln \alpha]^{2}}, & \alpha \neq 1,
\end{array}\right.\right.
$$




$$
\alpha=\left|\frac{f^{\prime}(b)}{f^{\prime}(a)}\right|^{s q / 2}
$$

and

$$
\begin{aligned}
& G_{1}\left(s, q ; g_{1}(\alpha), g_{2}(\alpha)\right) \\
& =\left\{\begin{array}{c}
\left|f^{\prime}(a)\right|^{s}\left[g_{1}(\alpha)\right]^{1 / q}+\left|f^{\prime}(a) f^{\prime}(b)\right|^{s / 2}\left[g_{2}(\alpha)\right]^{1 / q}, \quad\left|f^{\prime}(a)\right| \leq 1, \\
\left|f^{\prime}(a)\right|\left[g_{1}(\alpha)\right]^{1 / q}+\left|f^{\prime}(a)\right|^{1-s / 2}\left|f^{\prime}(b)\right|^{s / 2}\left[g_{2}(\alpha)\right]^{1 / q}, \quad\left|f^{\prime}(b)\right| \leq 1 \leq\left|f^{\prime}(a)\right|, \\
\left|f^{\prime}(a)\right|\left|f^{\prime}(b)\right|^{1-s}\left[g_{1}(\alpha)\right]^{1 / q}+\left|f^{\prime}(a) f^{\prime}(b)\right|^{1-s / 2}\left[g_{2}(\alpha)\right]^{1 / q}, \quad 1 \leq\left|f^{\prime}(b)\right| .
\end{array}\right.
\end{aligned}
$$

Theorem 1.2. [16] Let $f: I \subset \mathbb{R}_{+} \rightarrow \mathbb{R}$ be a differentiable on $I^{\circ}$ such that $f^{\prime} \in L([a, b])$ for $0<a<b<\infty$. If $\left|f^{\prime}(x)\right|^{q}$ is s-geometrically convex and monotonically decreasing on $[a, b]$ for $q>1$ and $s \in(0,1]$, then

$$
\begin{aligned}
& \left|f\left(\frac{a+b}{2}\right)-\frac{1}{b-a} \int_{a}^{b} f(x) d x\right| \leq \frac{b-a}{4}\left(\frac{q-1}{2 q-1}\right)^{1-1 / q} G_{2}\left(s, q ; g_{3}(\alpha)\right) \\
& \left|\frac{f(a)+f(b)}{2}-\frac{1}{b-a} \int_{a}^{b} f(x) d x\right| \leq \frac{b-a}{4}\left(\frac{q-1}{2 q-1}\right)^{1-1 / q} G_{2}\left(s, q ; g_{3}(\alpha)\right)
\end{aligned}
$$

where $\alpha$ is the same as in (1.12),

$$
g_{3}(\alpha)=\left\{\begin{array}{cc}
1, & \alpha=1 \\
\frac{\alpha-1}{\ln \alpha}, & \alpha \neq 1
\end{array}\right.
$$

and

$$
G_{2}\left(s, q ; g_{3}(\alpha)\right)=\left\{\begin{array}{c}
{\left[\left|f^{\prime}(a)\right|^{s}+\left|f^{\prime}(a) f^{\prime}(b)\right|^{s / 2}\right]\left[g_{3}(\alpha)\right]^{1 / q}, \quad\left|f^{\prime}(a)\right| \leq 1,} \\
{\left[\left|f^{\prime}(a)\right|+\left|f^{\prime}(a)\right|^{1-s / 2}\left|f^{\prime}(b)\right|^{s / 2}\right]\left[g_{3}(\alpha)\right]^{1 / q}, \quad\left|f^{\prime}(b)\right| \leq 1 \leq\left|f^{\prime}(a)\right|,} \\
\left(\left|f^{\prime}(a)\right|\left|f^{\prime}(b)\right|^{1-s}+\left|f^{\prime}(a) f^{\prime}(b)\right|^{1-s / 2}\right)\left[g_{3}(\alpha)\right]^{1 / q}, \quad 1 \leq\left|f^{\prime}(b)\right| .
\end{array}\right.
$$

\section{LemMas}

In order to prove our main theorems, we need the following lemmas.

Lemma 2.1. [14] Let $f: I \subseteq \mathbb{R} \rightarrow \mathbb{R}$ be differentiable function on $I^{\circ}, a, b \in I$, with $a<b$. If $f^{\prime} \in L[a, b]$ and $\lambda, \mu \in \mathbb{R}$ then

$$
\begin{aligned}
& \frac{\lambda f(a)+\mu f(b)}{2}+\frac{2-\lambda-\mu}{2} f\left(\frac{a+b}{2}\right)-\frac{1}{b-a} \int_{a}^{b} f(x) d x \\
= & \frac{b-a}{4} \int_{0}^{1}\left[(1-\lambda-t) f^{\prime}\left(t a+(1-t) \frac{a+b}{2}\right)+(\mu-t) f^{\prime}\left(t \frac{a+b}{2}+(1-t) b\right)\right] d t
\end{aligned}
$$

Lemma 2.2. [14] For $x>0$ and $0 \leq y \leq 1$, one has

$$
\begin{aligned}
\int_{0}^{1}|y-t|^{x} d t & =\frac{y^{x+1}+(1-y)^{x+1}}{x+1} \\
\int_{0}^{1} t|y-t|^{x} d t & =\frac{y^{x+2}+(x+1+y)(1-y)^{x+1}}{(x+1)(x+2)} .
\end{aligned}
$$


Lemma 2.3. [3] If $0<\varphi \leq 1 \leq \mu, 0<\alpha, \beta \leq 1$, then

$$
\varphi^{\alpha^{\beta}} \leq \varphi^{\alpha \beta} \text { and } \mu^{\alpha^{\beta}} \leq \mu^{\beta \alpha+1-\beta} .
$$

In this study, we will generalize the following theorems for $s$-geometrically convex functions by using the above three lemma and elementary analytical rules.

\section{General inequalities for $s$-Geometrically Convex functions}

Theorem 3.1. Let $f: I \subseteq \mathbb{R}_{+} \rightarrow \mathbb{R}_{+}$be differentiable on $I^{\circ}$, $a, b \in I$, with $a<b$, and $f^{\prime} \in L([a, b])$. If $\left|f^{\prime}(x)\right|$ is s-geometrically convex and monotonically decreasing on $[a, b]$, and $0<s \leq 1,0 \leq \lambda, \mu \leq 1$, then

$$
\begin{aligned}
& \left|\frac{\lambda f(a)+\mu f(b)}{2}+\frac{2-\lambda-\mu}{2} f\left(\frac{a+b}{2}\right)-\frac{1}{b-a} \int_{a}^{b} f(x) d x\right| \leq \frac{b-a}{4} \times \\
& \times\left\{\begin{array}{c}
\left|f^{\prime}(a) f^{\prime}(b)\right|^{\frac{s}{2}} M_{\lambda}(s ; Z)+\left|f^{\prime}(b)\right|^{s} E_{\mu}(s ; Z), \quad\left|f^{\prime}(a)\right| \leq 1 ; \\
\left.\left|f^{\prime}(a)\right|\right|^{1-\frac{s}{2}}\left|f^{\prime}(b)\right|^{\frac{s}{2}} M_{\lambda}(s ; Z)+\left|f^{\prime}(a)\right|^{1-s}\left|f^{\prime}(b)\right|^{s} E_{\mu}(s ; Z), \quad\left|f^{\prime}(b)\right| \leq 1 \leq\left|f^{\prime}(a)\right| ; \\
\left|f^{\prime}(a) f^{\prime}(b)\right|^{1-\frac{s}{2}} M_{\lambda}(s ; Z)+\left|f^{\prime}(a) f^{\prime}(b)\right|^{1-\frac{s}{2}} E_{\mu}(s ; Z), \quad 1 \leq\left|f^{\prime}(b)\right| ;
\end{array}\right.
\end{aligned}
$$

where

$$
\begin{aligned}
& M_{\lambda}(s ; Z)=\frac{\left(-1+\lambda+\lambda Z\left(\frac{s}{2}, \frac{s}{2}\right)\right) \ln Z\left(\frac{s}{2}, \frac{s}{2}\right)-1+2\left[Z\left(\frac{s}{2}, \frac{s}{2}\right)\right]^{1-\lambda}-Z\left(\frac{s}{2}, \frac{s}{2}\right)}{\left[\ln Z\left(\frac{s}{2}, \frac{s}{2}\right)\right]^{2}} \\
& E_{\mu}(s ; Z)=\frac{\left(-\mu-\mu Z\left(\frac{s}{2}, \frac{s}{2}\right)+Z\left(\frac{s}{2}, \frac{s}{2}\right)\right) \ln Z\left(\frac{s}{2}, \frac{s}{2}\right)-1+2\left[Z\left(\frac{s}{2}, \frac{s}{2}\right)\right]^{\mu}-Z\left(\frac{s}{2}, \frac{s}{2}\right)}{\left[\ln Z\left(\frac{s}{2}, \frac{s}{2}\right)\right]^{2}}
\end{aligned}
$$

and $Z(u, v)=\left|f^{\prime}(a)\right|^{u}\left|f^{\prime}(b)\right|^{-v}, u, v>0$.

Proof. From Lemma 2.1 and since $\left|f^{\prime}(x)\right|$ is $s$-geometrically convex and monotonically decreasing on $[a, b]$, we get

$$
\begin{aligned}
& \left|\frac{\lambda f(a)+\mu f(b)}{2}+\frac{2-\lambda-\mu}{2} f\left(\frac{a+b}{2}\right)-\frac{1}{b-a} \int_{a}^{b} f(x) d x\right| \\
\leq & \frac{b-a}{4}\left[\int_{0}^{1}|1-\lambda-t|\left|f^{\prime}\left(t a+(1-t) \frac{a+b}{2}\right)\right| d t+\int_{0}^{1}|\mu-t|\left|f^{\prime}\left(t \frac{a+b}{2}+(1-t) b\right)\right| d t\right] \\
= & \frac{b-a}{4}\left[\int_{0}^{1}|1-\lambda-t|\left|f^{\prime}\left(\frac{1+t}{2} a+\frac{1-t}{2} b\right)\right| d t+\int_{0}^{1}|\mu-t|\left|f^{\prime}\left(\frac{t}{2} a+\frac{2-t}{2} b\right)\right| d t\right] \\
\leq & \frac{b-a}{4}\left[\int_{0}^{1}|1-\lambda-t|\left|f^{\prime}\left(a^{\frac{1+t}{2}} b^{\frac{1-t}{2}}\right)\right| d t+\int_{0}^{1}|\mu-t|\left|f^{\prime}\left(a^{\frac{t}{2}} b^{\frac{2-t}{2}}\right)\right| d t\right] \\
\leq & \frac{b-a}{4}\left[\int_{0}^{1}|1-\lambda-t|\left|f^{\prime}(a)\right|^{\left(\frac{1+t}{2}\right)^{s}}\left|f^{\prime}(b)\right|^{\left(\frac{1-t}{2}\right)^{s}} d t+\int_{0}^{1}|\mu-t|\left|f^{\prime}(a)\right|^{\left(\frac{t}{2}\right)^{s}}\left|f^{\prime}(b)\right|^{\left(\frac{2-t}{2}\right)^{s}} d t\right] .
\end{aligned}
$$


When $\left|f^{\prime}(a)\right| \leq 1$, by using (2.3), we get

$$
\begin{aligned}
& \left|\frac{\lambda f(a)+\mu f(b)}{2}+\frac{2-\lambda-\mu}{2} f\left(\frac{a+b}{2}\right)-\frac{1}{b-a} \int_{a}^{b} f(x) d x\right| \\
\leq & \frac{b-a}{4}\left[\int_{0}^{1}|1-\lambda-t|\left|f^{\prime}(a)\right|^{\left(\frac{1+t}{2}\right)^{s}}\left|f^{\prime}(b)\right|^{\left(\frac{1-t}{2}\right)^{s}} d t+\int_{0}^{1}|\mu-t|\left|f^{\prime}(a)\right|^{\left(\frac{t}{2}\right)^{s}}\left|f^{\prime}(b)\right|^{\left(\frac{2-t}{2}\right)^{s}} d t\right] \\
\leq & \frac{b-a}{4}\left[\int_{0}^{1}|1-\lambda-t|\left|f^{\prime}(a)\right|^{\frac{s+s t}{2}}\left|f^{\prime}(b)\right|^{\frac{s-s t}{2}} d t+\int_{0}^{1}|\mu-t|\left|f^{\prime}(a)\right|^{\frac{s t}{2}}\left|f^{\prime}(b)\right|^{\frac{2 s-s t}{2}} d t\right] \\
= & \frac{b-a}{4}\left[\left|f^{\prime}(a) f^{\prime}(b)\right|^{\frac{s}{2}} \int_{0}^{1}|1-\lambda-t|\left|f^{\prime}(a) / f^{\prime}(b)\right|^{\frac{s t}{2}} d t+\left|f^{\prime}(b)\right|^{s}|\mu-t|\left|f^{\prime}(a) / f^{\prime}(b)\right|^{\frac{s t}{2}} d t\right]
\end{aligned}
$$

In a direct calculation yields,

$$
\begin{aligned}
& \int_{0}^{1}|1-\lambda-t|\left|f^{\prime}(a) / f^{\prime}(b)\right|^{\frac{s t}{2}} d t \\
= & \frac{\left(\left(-1+\lambda+\lambda Z\left(\frac{s}{2}, \frac{s}{2}\right)\right) \ln Z\left(\frac{s}{2}, \frac{s}{2}\right)-1+2\left[Z\left(\frac{s}{2}, \frac{s}{2}\right)\right]^{1-\lambda}-Z\left(\frac{s}{2}, \frac{s}{2}\right)\right)}{\left[\ln Z\left(\frac{s}{2}, \frac{s}{2}\right)\right]^{2}} \\
= & M_{\lambda}(s ; Z)
\end{aligned}
$$

and

$$
\begin{aligned}
& \int_{0}^{1}|\mu-t|\left|f^{\prime}(a) / f^{\prime}(b)\right|^{\frac{s t}{2}} d t \\
= & \frac{\left(\left(-\mu-\mu Z\left(\frac{s}{2}, \frac{s}{2}\right)+Z\left(\frac{s}{2}, \frac{s}{2}\right)\right) \ln Z\left(\frac{s}{2}, \frac{s}{2}\right)-1+2\left[Z\left(\frac{s}{2}, \frac{s}{2}\right)\right]^{\mu}-Z\left(\frac{s}{2}, \frac{s}{2}\right)\right)}{\left[\ln Z\left(\frac{s}{2}, \frac{s}{2}\right)\right]^{2}} \\
= & E_{\mu}(s ; Z)
\end{aligned}
$$

If (3.2) is rewritten, we have

$$
\begin{aligned}
& \left|\frac{\lambda f(a)+\mu f(b)}{2}+\frac{2-\lambda-\mu}{2} f\left(\frac{a+b}{2}\right)-\frac{1}{b-a} \int_{a}^{b} f(x) d x\right| \\
\leq & \frac{b-a}{4}\left[\left|f^{\prime}(a) f^{\prime}(b)\right|^{\frac{s}{2}} M_{\lambda}(s ; Z)+\left|f^{\prime}(b)\right|^{s} E_{\mu}(s ; Z)\right]
\end{aligned}
$$

When $\left|f^{\prime}(b)\right| \leq 1 \leq\left|f^{\prime}(a)\right|$, from (2.3), we get

$$
\begin{aligned}
& \left|\frac{\lambda f(a)+\mu f(b)}{2}+\frac{2-\lambda-\mu}{2} f\left(\frac{a+b}{2}\right)-\frac{1}{b-a} \int_{a}^{b} f(x) d x\right| \\
\leq & \frac{b-a}{4}\left[\int_{0}^{1}|1-\lambda-t|\left|f^{\prime}(a)\right|^{\left(\frac{1+t}{2}\right)^{s}}\left|f^{\prime}(b)\right|^{\left(\frac{1-t}{2}\right)^{s}} d t+\int_{0}^{1}|\mu-t|\left|f^{\prime}(a)\right|^{\left(\frac{t}{2}\right)^{s}}\left|f^{\prime}(b)\right|^{\left(\frac{2-t}{2}\right)^{s}} d t\right] \\
\leq & \frac{b-a}{4}\left[\int_{0}^{1}|1-\lambda-t|\left|f^{\prime}(a)\right|^{\frac{s+s t}{2}+1-s}\left|f^{\prime}(b)\right|^{\frac{s-s t}{2}} d t+\int_{0}^{1}|\mu-t|\left|f^{\prime}(a)\right|^{\frac{s t}{2}+1-s}\left|f^{\prime}(b)\right|^{\frac{2 s-s t}{2}} d t\right] \\
= & \frac{b-a}{4}\left[\left|f^{\prime}(a)\right|^{1-\frac{s}{2}}\left|f^{\prime}(b)\right|^{\frac{s}{2}} M_{\lambda}(s ; Z)+\left|f^{\prime}(a)\right|^{1-s}\left|f^{\prime}(b)\right|^{s} E_{\mu}(s ; Z)\right]
\end{aligned}
$$


When $1 \leq\left|f^{\prime}(b)\right|$, by virtue of (2.3), we get

$$
\begin{aligned}
& \left|\frac{\lambda f(a)+\mu f(b)}{2}+\frac{2-\lambda-\mu}{2} f\left(\frac{a+b}{2}\right)-\frac{1}{b-a} \int_{a}^{b} f(x) d x\right| \\
& \leq \frac{b-a}{4}\left[\int_{0}^{1}|1-\lambda-t|\left|f^{\prime}(a)\right|^{\left(\frac{1+t}{2}\right)^{s}}\left|f^{\prime}(b)\right|^{\left(\frac{1-t}{2}\right)^{s}} d t\right. \\
& \left.+\int_{0}^{1}|\mu-t|\left|f^{\prime}(a)\right|^{\left(\frac{t}{2}\right)^{s}}\left|f^{\prime}(b)\right|^{\left(\frac{2-t}{2}\right)^{s}} d t\right] \\
& \leq \frac{b-a}{4}\left[\int_{0}^{1}|1-\lambda-t|\left|f^{\prime}(a)\right|^{\frac{s+s t}{2}+1-s}\left|f^{\prime}(b)\right|^{\frac{s-s t}{2}+1-s} d t\right. \\
& \left.+\int_{0}^{1}|\mu-t|\left|f^{\prime}(a)\right|^{\frac{s t}{2}+1-s}\left|f^{\prime}(b)\right|^{\frac{2 s-s t}{2}+1-s} d t\right] \\
& =\frac{b-a}{4}\left[\left|f^{\prime}(a) f^{\prime}(b)\right|^{1-\frac{s}{2}} M_{\lambda}(s ; Z)+\left|f^{\prime}(a) f^{\prime}(b)\right|^{1-\frac{s}{2}} E_{\mu}(s ; Z)\right] \text {. }
\end{aligned}
$$

The proof of Theorem 3.1 is complete.

If taking $\lambda=\mu$ in Theorem 3.1, we derive the following corollary.

Corollary 3.1. Let $f: I \subseteq \mathbb{R}_{+} \rightarrow \mathbb{R}_{+}$be differentiable on $I^{\circ}$, a,b $\in I$, with $a<b$, and $f^{\prime} \in L([a, b])$. If $\left|f^{\prime}(x)\right|$ is s-geometrically convex and monotonically decreasing on $[a, b]$, and $0<s \leq 1,0 \leq \lambda \leq 1$, then

$$
\begin{aligned}
& \left|\frac{\lambda[f(a)+f(b)]}{2}+(1-\lambda) f\left(\frac{a+b}{2}\right)-\frac{1}{b-a} \int_{a}^{b} f(x) d x\right| \leq \frac{b-a}{4} \\
& \times\left\{\begin{array}{c}
\left|f^{\prime}(a) f^{\prime}(b)\right|^{\frac{s}{2}} M(\lambda, s ; Z)+\left|f^{\prime}(b)\right|^{s} E(\lambda, s ; Z), \quad\left|f^{\prime}(a)\right| \leq 1 ; \\
\left|f^{\prime}(a)\right|^{1-\frac{s}{2}}\left|f^{\prime}(b)\right|^{\frac{3}{2}} M(\lambda, s ; Z)+\left|f^{\prime}(a)\right|^{1-s}\left|f^{\prime}(b)\right|^{s} E(\lambda, s ; Z), \quad\left|f^{\prime}(b)\right| \leq 1 \leq\left|f^{\prime}(a)\right| ; \\
\left|f^{\prime}(a) f^{\prime}(b)\right|^{1-\frac{s}{2}} M(\lambda, s ; Z)+\left|f^{\prime}(a) f^{\prime}(b)\right|^{1-\frac{s}{2}} E(\lambda, s ; Z), \quad 1 \leq\left|f^{\prime}(b)\right| ;
\end{array}\right.
\end{aligned}
$$

where $Z(u, v), M_{\lambda}(s ; Z), E_{\lambda}(s ; Z)$ are defined in Theorem 3.1.

If letting $\lambda=\mu=1 / 2,2 / 3,1 / 3$, respectively, in Theorem 3.1, we can deduce the inequalities below.

Corollary 3.2. Let $f: I \subseteq \mathbb{R}_{+} \rightarrow \mathbb{R}_{+}$be differentiable on $I^{\circ}, a, b \in I$, with $a<b$, and $f^{\prime} \in L([a, b])$. If $\left|f^{\prime}(x)\right|$ is s-geometrically convex and monotonically decreasing on $[a, b]$, and $0<s \leq 1$, then

$$
\begin{aligned}
& \left|\frac{1}{2}\left[\frac{f(a)+f(b)}{2}+f\left(\frac{a+b}{2}\right)\right]-\frac{1}{b-a} \int_{a}^{b} f(x) d x\right| \leq \frac{b-a}{4} \\
& \times\left\{\begin{array}{c}
\left|f^{\prime}(a) f^{\prime}(b)\right|^{\frac{s}{2}} M_{1 / 2}(s ; Z)+\left|f^{\prime}(b)\right|^{s} E_{1 / 2}(s ; Z), \quad\left|f^{\prime}(a)\right| \leq 1 ; \\
\left|f^{\prime}(a)\right|^{1-\frac{s}{2}}\left|f^{\prime}(b)\right|^{\frac{s}{2}} M_{1 / 2}(s ; Z)+\left|f^{\prime}(a)\right|^{1-s}\left|f^{\prime}(b)\right|^{s} E_{1 / 2}(s ; Z), \quad\left|f^{\prime}(b)\right| \leq 1 \leq\left|f^{\prime}(a)\right| ; \\
\left|f^{\prime}(a) f^{\prime}(b)\right|^{1-\frac{s}{2}} M_{1 / 2}(s ; Z)+\left|f^{\prime}(a) f^{\prime}(b)\right|^{1-\frac{s}{2}} E_{1 / 2}(s ; Z), \quad 1 \leq\left|f^{\prime}(b)\right| ;
\end{array}\right.
\end{aligned}
$$




$$
\begin{aligned}
& \left|\frac{1}{3}\left[f(a)+f(b)+f\left(\frac{a+b}{2}\right)\right]-\frac{1}{b-a} \int_{a}^{b} f(x) d x\right| \leq \frac{b-a}{4} \\
& \times\left\{\begin{array}{c}
\left|f^{\prime}(a) f^{\prime}(b)\right|^{\frac{s}{2}} M_{2 / 3}(s ; Z)+\left|f^{\prime}(b)\right|^{s} E_{2 / 3}(s ; Z), \quad\left|f^{\prime}(a)\right| \leq 1 ; \\
\left|f^{\prime}(a)\right|^{1-\frac{s}{2}}\left|f^{\prime}(b)\right| \frac{s}{2} M_{2 / 3}(s ; Z)+\left|f^{\prime}(a)\right|^{1-s}\left|f^{\prime}(b)\right|^{s} E_{2 / 3}(s ; Z), \quad\left|f^{\prime}(b)\right| \leq 1 \leq\left|f^{\prime}(a)\right| ; \\
\left|f^{\prime}(a) f^{\prime}(b)\right|^{1-\frac{s}{2}} M_{2 / 3}(s ; Z)+\left|f^{\prime}(a) f^{\prime}(b)\right|^{1-\frac{s}{2}} E_{2 / 3}(s ; Z), \quad 1 \leq\left|f^{\prime}(b)\right| ;
\end{array}\right.
\end{aligned}
$$

$\left|\frac{1}{6}\left[f(a)+f(b)+4 f\left(\frac{a+b}{2}\right)\right]-\frac{1}{b-a} \int_{a}^{b} f(x) d x\right| \leq \frac{b-a}{4}$

$$
\times\left\{\begin{array}{c}
\left|f^{\prime}(a) f^{\prime}(b)\right|^{\frac{s}{2}} M_{1 / 3}(s ; Z)+\left|f^{\prime}(b)\right|^{s} E_{1 / 3}(s ; Z), \quad\left|f^{\prime}(a)\right| \leq 1 ; \\
\left|f^{\prime}(a)\right|^{1-\frac{s}{2}}\left|f^{\prime}(b)\right|^{\frac{s}{2}} M_{1 / 3}(s ; Z)+\left|f^{\prime}(a)\right|^{1-s}\left|f^{\prime}(b)\right|^{s} E_{1 / 3}(s ; Z), \quad\left|f^{\prime}(b)\right| \leq 1 \leq\left|f^{\prime}(a)\right| ; \\
\left|f^{\prime}(a) f^{\prime}(b)\right|^{1-\frac{s}{2}} M_{1 / 3}(s ; Z)+\left|f^{\prime}(a) f^{\prime}(b)\right|^{1-\frac{s}{2}} E_{1 / 3}(s ; Z), \quad 1 \leq\left|f^{\prime}(b)\right|
\end{array}\right.
$$

where $Z(u, v), M_{\lambda}(s ; Z), E_{\mu}(s ; Z)$ are defined in Theorem 3.1.

Theorem 3.2. Let $f: I \subseteq \mathbb{R}_{+} \rightarrow \mathbb{R}_{+}$be differentiable on $I^{\circ}, a, b \in I$ with $a<b$, $0 \leq \lambda, \mu \leq 1$, and $f^{\prime}$ is integrable on $[a, b]$. If $\left|f^{\prime}(x)\right|^{q}$ is s-geometrically convex and monotonically decreasing on $[a, b]$, for $q \geq 1$ and $s \in(0,1]$, then

$$
\begin{aligned}
& \left|\frac{\lambda f(a)+\mu f(b)}{2}+\frac{2-\lambda-\mu}{2} f\left(\frac{a+b}{2}\right)-\frac{1}{b-a} \int_{a}^{b} f(x) d x\right| \leq \frac{b-a}{4} \times \\
& \left\{\begin{array}{c}
\left|f^{\prime}(a) f^{\prime}(b)\right|^{\frac{s}{2}}\left(\frac{(1-\lambda)^{2}+\lambda^{2}}{2}\right)^{1-\frac{1}{q}} M_{\lambda}^{1 / q}(s, q ; Z) \\
\quad+\left|f^{\prime}(a) f^{\prime}(b)\right|^{s}\left(\frac{\mu^{2}+(1-\mu)^{2}}{2}\right)^{1-\frac{1}{q}} E_{\mu}^{1 / q}(s, q ; Z) \\
\left|f^{\prime}(a)\right|^{1-\frac{s}{2}}\left|f^{\prime}(b)\right|^{\frac{s}{2}}\left(\frac{(1-\lambda)^{2}+\lambda^{2}}{2}\right)^{1-\frac{1}{q}} M_{\lambda}^{1 / q}(s, q ; Z) \\
+\left|f^{\prime}(a)\right|^{1-s}\left|f^{\prime}(b)\right|^{s}\left(\frac{\mu^{2}+(1-\mu)^{2}}{2}\right)^{1-\frac{1}{q}} E_{\mu}^{1 / q}(s, q ; Z) \\
\left|f^{\prime}(a) f^{\prime}(b)\right|^{1-\frac{s}{2}}\left(\frac{(1-\lambda)^{2}+\lambda^{2}}{2}\right)^{1-\frac{1}{q}} M_{\lambda}^{1 / q}(s, q ; Z) \\
+\left|f^{\prime}(a)\right|^{1-s}\left|f^{\prime}(b)\right|\left(\frac{\mu^{2}+(1-\mu)^{2}}{2}\right)^{1-\frac{1}{q}} E_{\mu}^{1 / q}(s, q ; Z)
\end{array} \quad, \quad\left|f^{\prime}(b)\right| \leq 1 \leq\left|f^{\prime}(a)\right| ;\right.
\end{aligned}
$$

where

$$
\begin{aligned}
& M_{\lambda}(s, q ; Z) \\
= & \frac{\left(-1+\lambda+\lambda Z\left(\frac{s q}{2}, \frac{s q}{2}\right)\right) \ln Z\left(\frac{s q}{2}, \frac{s q}{2}\right)-1+2\left[Z\left(\frac{s q}{2}, \frac{s q}{2}\right)\right]^{1-\lambda}-Z\left(\frac{s q}{2}, \frac{s q}{2}\right)}{\left[\ln Z\left(\frac{s q}{2}, \frac{s q}{2}\right)\right]^{2}} \\
& E_{\mu}(s, q ; Z) \\
= & \frac{\left(-\mu-\mu Z\left(\frac{s q}{2}, \frac{s q}{2}\right)+Z\left(\frac{s q}{2}, \frac{s q}{2}\right)\right) \ln Z\left(\frac{s q}{2}, \frac{s q}{2}\right)-1+2\left[Z\left(\frac{s q}{2}, \frac{s q}{2}\right)\right]^{\mu}-Z\left(\frac{s q}{2}, \frac{s q}{2}\right)}{\left[\ln Z\left(\frac{s q}{2}, \frac{s q}{2}\right)\right]^{2}}
\end{aligned}
$$

and $Z(u, v)=\left|f^{\prime}(a)\right|^{u}\left|f^{\prime}(b)\right|^{-v}, u, v>0$. 
Proof. From Lemma 2.1 and since $\left|f^{\prime}(x)\right|^{q}$ is $s$-geometrically convex and monotonically decreasing on $[a, b]$, by using well known power mean inequality, we get

$$
\begin{aligned}
& \left|\frac{\lambda f(a)+\mu f(b)}{2}+\frac{2-\lambda-\mu}{2} f\left(\frac{a+b}{2}\right)-\frac{1}{b-a} \int_{a}^{b} f(x) d x\right| \\
\leq & \frac{b-a}{4}\left[\left(\int_{0}^{1}|1-\lambda-t| d t\right)^{1-\frac{1}{q}}\left(\int_{0}^{1}|1-\lambda-t|\left(\left|f^{\prime}\left(a^{\frac{1+t}{2}} b^{\frac{1-t}{2}}\right)\right|\right)^{q} d t\right)^{\frac{1}{q}}\right. \\
& \left.+\left(\int_{0}^{1}|\mu-t| d t\right)^{1-\frac{1}{q}}\left(\int_{0}^{1}|\mu-t|\left(\left|f^{\prime}\left(a^{\frac{t}{2}} b^{\frac{2-t}{2}}\right)\right|\right)^{q} d t\right)^{\frac{1}{q}}\right] \\
\leq & \frac{b-a}{4}\left[\left(\frac{(1-\lambda)^{2}+\lambda^{2}}{2}\right)^{1-\frac{1}{q}}\left(\int_{0}^{1}|1-\lambda-t|\left|f^{\prime}(a)\right|^{q\left(\frac{1+t}{2}\right)^{s}}\left|f^{\prime}(b)\right|^{q\left(\frac{1-t}{2}\right)^{s}} d t\right)^{\frac{1}{q}}\right. \\
& \left.+\left(\frac{\mu^{2}+(1-\mu)^{2}}{2}\right)^{1-\frac{1}{q}}\left(\int_{0}^{1}|\mu-t|\left|f^{\prime}(a)\right|^{q\left(\frac{t}{2}\right)^{s}}\left|f^{\prime}(b)\right|^{q\left(\frac{2-t}{2}\right)^{s}} d t\right)^{\frac{1}{q}}\right] .
\end{aligned}
$$

When $\left|f^{\prime}(a)\right| \leq 1$, by $(2.3)$, we get

$$
\begin{aligned}
& \int_{0}^{1}|1-\lambda-t|\left|f^{\prime}(a)\right|^{q\left(\frac{1+t}{2}\right)^{s}}\left|f^{\prime}(b)\right|^{q\left(\frac{1-t}{2}\right)^{s}} d t \\
\leq & \int_{0}^{1}|1-\lambda-t|\left|f^{\prime}(a)\right|^{s q\left(\frac{1+t}{2}\right)}\left|f^{\prime}(b)\right|^{s q\left(\frac{1-t}{2}\right)} d t \\
= & \left|f^{\prime}(a) f^{\prime}(b)\right|^{\frac{s q}{2}} \int_{0}^{1}|1-\lambda-t|\left|f^{\prime}(a) / f^{\prime}(b)\right|^{\frac{s q t}{2}} d t \\
= & \left|f^{\prime}(a) f^{\prime}(b)\right|^{\frac{s q}{2}} \\
& \times \frac{\left(-1+\lambda+\lambda Z\left(\frac{s q}{2}, \frac{s q}{2}\right)\right) \ln Z\left(\frac{s q}{2}, \frac{s q}{2}\right)-1+2\left[Z\left(\frac{s q}{2}, \frac{s q}{2}\right)\right]^{1-\lambda}-Z\left(\frac{s q}{2}, \frac{s q}{2}\right)}{\left[\ln Z\left(\frac{s q}{2}, \frac{s q}{2}\right)\right]^{2}} \\
= & \left|f^{\prime}(a) f^{\prime}(b)\right|^{\frac{s q}{2}} M_{\lambda}(s, q ; Z)
\end{aligned}
$$

and

$$
\begin{aligned}
& \int_{0}^{1}|\mu-t|\left|f^{\prime}(a)\right|^{q\left(\frac{t}{2}\right)^{s}}\left|f^{\prime}(b)\right|^{q\left(\frac{2-t}{2}\right)^{s}} d t \\
\leq & \int_{0}^{1}|\mu-t|\left|f^{\prime}(a)\right|^{s q\left(\frac{t}{2}\right)}\left|f^{\prime}(b)\right|^{s q\left(\frac{2-t}{2}\right)} d t \\
= & \left|f^{\prime}(a) f^{\prime}(b)\right|^{s q} \int_{0}^{1}|\mu-t|\left|f^{\prime}(a) / f^{\prime}(b)\right|^{\frac{s q t}{2}} d t \\
= & \left|f^{\prime}(a) f^{\prime}(b)\right|^{s q} \\
& \times \frac{\left(-\mu-\mu Z\left(\frac{s q}{2}, \frac{s q}{2}\right)+Z\left(\frac{s q}{2}, \frac{s q}{2}\right)\right) \ln Z\left(\frac{s q}{2}, \frac{s q}{2}\right)-1+2\left[Z\left(\frac{s q}{2}, \frac{s q}{2}\right)\right]^{\mu}-Z\left(\frac{s q}{2}, \frac{s q}{2}\right)}{\left[\ln Z\left(\frac{s q}{2}, \frac{s q}{2}\right)\right]^{2}} \\
= & \left|f^{\prime}(a) f^{\prime}(b)\right|^{s q} E_{\mu}(s, q ; Z) .
\end{aligned}
$$


When $\left|f^{\prime}(b)\right| \leq 1 \leq\left|f^{\prime}(a)\right|$, by (2.3), we get

$$
\begin{aligned}
& \int_{0}^{1}|1-\lambda-t|\left|f^{\prime}(a)\right|^{q\left(\frac{1+t}{2}\right)^{s}}\left|f^{\prime}(b)\right|^{q\left(\frac{1-t}{2}\right)^{s}} d t \\
\leq & \int_{0}^{1}|1-\lambda-t|\left|f^{\prime}(a)\right|^{q\left[s\left(\frac{1+t}{2}\right)+1-s\right]}\left|f^{\prime}(b)\right|^{s q\left(\frac{1-t}{2}\right)} d t \\
= & \left|f^{\prime}(a)\right|^{q\left(1-\frac{s}{2}\right)}\left|f^{\prime}(b)\right|^{\frac{s q}{2}} \int_{0}^{1}|1-\lambda-t|\left|f^{\prime}(a) / f^{\prime}(b)\right|^{\frac{s q t}{2}} d t \\
= & \left|f^{\prime}(a)\right|^{q\left(1-\frac{s}{2}\right)}\left|f^{\prime}(b)\right|^{\frac{s q}{2}} M_{\lambda}(s, q ; Z)
\end{aligned}
$$

and

$$
\begin{aligned}
& \int_{0}^{1}|\mu-t|\left|f^{\prime}(a)\right|^{q\left(\frac{t}{2}\right)^{s}}\left|f^{\prime}(b)\right|^{q\left(\frac{2-t}{2}\right)^{s}} d t \\
\leq & \int_{0}^{1}|\mu-t|\left|f^{\prime}(a)\right|^{q\left[s\left(\frac{t}{2}\right)+1-s\right]}\left|f^{\prime}(b)\right|^{s q\left(\frac{2-t}{2}\right)} d t \\
= & \left|f^{\prime}(a)\right|^{q(1-s)}\left|f^{\prime}(b)\right|^{s q} \int_{0}^{1}|\mu-t|\left|f^{\prime}(a) / f^{\prime}(b)\right|^{\frac{s q t}{2}} d t \\
= & \left|f^{\prime}(a)\right|^{q(1-s)}\left|f^{\prime}(b)\right|^{s q} E_{\mu}(s, q ; Z) .
\end{aligned}
$$

When $1 \leq\left|f^{\prime}(b)\right|$, by $(2.3)$, we get

$$
\begin{aligned}
& \int_{0}^{1}|1-\lambda-t|\left|f^{\prime}(a)\right|^{q\left(\frac{1+t}{2}\right)^{s}}\left|f^{\prime}(b)\right|^{q\left(\frac{1-t}{2}\right)^{s}} d t \\
\leq & \int_{0}^{1}|1-\lambda-t|\left|f^{\prime}(a)\right|^{q\left[s\left(\frac{1+t}{2}\right)+1-s\right]}\left|f^{\prime}(b)\right|^{q\left[s\left(\frac{1-t}{2}\right)+1-s\right]} d t \\
= & \left|f^{\prime}(a) f^{\prime}(b)\right|^{q\left(1-\frac{s}{2}\right)} \int_{0}^{1}|1-\lambda-t|\left|f^{\prime}(a) / f^{\prime}(b)\right|^{\frac{s q t}{2}} d t \\
= & \left|f^{\prime}(a) f^{\prime}(b)\right|^{q\left(1-\frac{s}{2}\right)} M_{\lambda}(s, q ; Z)
\end{aligned}
$$

and

$$
\begin{aligned}
& \int_{0}^{1}|\mu-t|\left|f^{\prime}(a)\right|^{q\left(\frac{t}{2}\right)^{s}}\left|f^{\prime}(b)\right|^{q\left(\frac{2-t}{2}\right)^{s}} d t \\
\leq & \int_{0}^{1}|\mu-t|\left|f^{\prime}(a)\right|^{q\left[\frac{s t}{2}+1-s\right]}\left|f^{\prime}(b)\right|^{q\left[s\left(\frac{2-t}{2}\right)+1-s\right]} d t \\
= & \left|f^{\prime}(a)\right|^{q(1-s)}\left|f^{\prime}(b)\right|^{q} \int_{0}^{1}|\mu-t|\left|f^{\prime}(a) / f^{\prime}(b)\right|^{\frac{s q t}{2}} d t \\
= & \left|f^{\prime}(a)\right|^{q(1-s)}\left|f^{\prime}(b)\right|^{q} E_{\mu}(s, q ; Z) .
\end{aligned}
$$

As a result, the proof of Theorem 3.2 is complete.

If taking $\lambda=\mu$ in Theorem 3.2, we derive the following corollary.

Corollary 3.3. Let $f: I \subseteq \mathbb{R}_{+} \rightarrow \mathbb{R}_{+}$be differentiable on $I^{\circ}, a, b \in I$, with $a<b$, and $f^{\prime} \in L([a, b])$. If $\left|f^{\prime}(x)\right|^{q}$ is s-geometrically convex and monotonically 
decreasing on $[a, b]$, for $q \geq 1$ and $0<s \leq 1,0 \leq \lambda \leq 1$, then

$$
\begin{aligned}
& \left|\frac{\lambda f(a)+\mu f(b)}{2}+\frac{2-\lambda-\mu}{2} f\left(\frac{a+b}{2}\right)-\frac{1}{b-a} \int_{a}^{b} f(x) d x\right| \\
\leq & \frac{b-a}{4}\left(\frac{(1-\lambda)^{2}+\lambda^{2}}{2}\right)^{1-\frac{1}{q}} \times \\
& \left\{\begin{array}{c}
\left|f^{\prime}(a) f^{\prime}(b)\right|^{\frac{s}{2}} M_{\lambda}^{1 / q}(s, q ; Z) \\
+\left|f^{\prime}(a) f^{\prime}(b)\right|^{s} E_{\lambda}^{1 / q}(s, q ; Z) \\
\left|f^{\prime}(a)\right|^{1-\frac{s}{2}}\left|f^{\prime}(b)\right|^{\frac{s}{2}} M_{\lambda}^{1 / q}(s, q ; Z) \\
+\left|f^{\prime}(a)\right|^{1-s}\left|f^{\prime}(b)\right|^{s} E_{\lambda}^{1 / q}(s, q ; Z) \\
\left|f^{\prime}(a) f^{\prime}(b)\right|^{1-\frac{s}{2}} M_{\lambda}^{1 / q}(s, q ; Z) \\
+\left|f^{\prime}(a)\right|^{1-s}\left|f^{\prime}(b)\right| E_{\lambda}^{1 / q}(s, q ; Z)
\end{array}, \quad\left|f^{\prime}(b)\right| \leq 1 \leq\left|f^{\prime}(a)\right| ;\right. \\
&
\end{aligned}
$$

where $Z(u, v), M_{\lambda}(s, q ; Z), E_{\mu}(s, q ; Z)$ are defined in Theorem 3.2.

If letting $\lambda=\mu=1 / 2,2 / 3,1 / 3$, respectively, in Theorem 3.2 , we can deduce the inequalities below.

Corollary 3.4. Let $f: I \subseteq \mathbb{R}_{+} \rightarrow \mathbb{R}_{+}$be differentiable on $I^{\circ}, a, b \in I$, with $a<b$, and $f^{\prime} \in L([a, b])$. If $\left|f^{\prime}(x)\right|^{q}$ is s-geometrically convex and monotonically decreasing on $[a, b]$, for $q \geq 1$ and $0<s \leq 1$, then

$$
\begin{aligned}
& \left|\frac{1}{2}\left[\frac{f(a)+f(b)}{2}+f\left(\frac{a+b}{2}\right)\right]-\frac{1}{b-a} \int_{a}^{b} f(x) d x\right| \\
& \leq \frac{4^{1 / q}(b-a)}{16} \times \\
& \left\{\begin{aligned}
\left|f^{\prime}(a) f^{\prime}(b)\right|^{\frac{s}{2}} M_{1 / 2}^{1 / q}(s, q ; Z) & \\
+\left|f^{\prime}(a) f^{\prime}(b)\right|^{s} E_{1 / 2}^{1 / q}(s, q ; Z), & \left|f^{\prime}(a)\right| \leq 1 ; \\
\left|f^{\prime}(a)\right|^{1-\frac{s}{2}}\left|f^{\prime}(b)\right|^{\frac{s}{2}} M_{1 / 2}^{1 / q}(s, q ; Z) & \\
+\left|f^{\prime}(a)\right|^{1-s}\left|f^{\prime}(b)\right|^{s} E_{1 / 2}^{1 / q}(s, q ; Z), &
\end{aligned}\right. \\
& \left|f^{\prime}(a) f^{\prime}(b)\right|^{1-\frac{s}{2}} M_{1 / 2}^{1 / q}(s, q ; Z) \\
& +\left|f^{\prime}(a)\right|^{1-s}\left|f^{\prime}(b)\right| E_{1 / 2}^{1 / 2}(s, q ; Z), \quad, \quad 1 \leq\left|f^{\prime}(b)\right| ; \\
& \left|\frac{1}{3}\left[f(a)+f(b)+f\left(\frac{a+b}{2}\right)\right]-\frac{1}{b-a} \int_{a}^{b} f(x) d x\right| \\
& \leq \frac{5(b-a)}{72}\left(\frac{18}{5}\right)^{1 / q} \times \\
& \left\{\begin{array}{c}
\left|f^{\prime}(a) f^{\prime}(b)\right|^{\frac{s}{2}} M_{2 / 3}^{1 / q}(s, q ; Z)+\left|f^{\prime}(a) f^{\prime}(b)\right|^{s} E_{2 / 3}^{1 / q}(s, q ; Z), \quad\left|f^{\prime}(a)\right| \leq 1 ; \\
\left|f^{\prime}(a)\right|^{1-\frac{s}{2}}\left|f^{\prime}(b)\right|^{\frac{s}{2}} M_{2 / 3}^{1 / q}(s, q ; Z)+\left|f^{\prime}(a)\right|^{1-s}\left|f^{\prime}(b)\right|^{s} E_{2 / 3}^{1 / q}(s, q ; Z), \quad\left|f^{\prime}(b)\right| \leq 1 \leq\left|f^{\prime}(a)\right| ; \\
\left|f^{\prime}(a) f^{\prime}(b)\right|^{1-\frac{s}{2}} M_{2 / 3}^{1 / q}(s, q ; Z)+\left|f^{\prime}(a)\right|^{1-s}\left|f^{\prime}(b)\right| E_{2 / 3}^{1 / q}(s, q ; Z), \quad 1 \leq\left|f^{\prime}(b)\right| ;
\end{array}\right.
\end{aligned}
$$




$$
\begin{aligned}
& \left|\frac{1}{6}\left[f(a)+f(b)+4 f\left(\frac{a+b}{2}\right)\right]-\frac{1}{b-a} \int_{a}^{b} f(x) d x\right| \\
\leq & \frac{5(b-a)}{72}\left(\frac{18}{5}\right)^{1 / q} \times \\
& \left\{\begin{array}{r}
\left|f^{\prime}(a) f^{\prime}(b)\right|^{\frac{s}{2}} M_{1 / 3}^{1 / q}(s, q ; Z)+\left|f^{\prime}(a) f^{\prime}(b)\right|^{s} E_{1 / 3}^{1 / q}(s, q ; Z),\left|f^{\prime}(a)\right| \leq 1 ; \\
\left|f^{\prime}(a)\right|^{1-\frac{s}{2}}\left|f^{\prime}(b)\right|^{\frac{s}{2}} M_{1 / 3}^{1 / q}(s, q ; Z)+\left|f^{\prime}(a)\right|^{1-s}\left|f^{\prime}(b)\right|^{s} E_{1 / 3}^{1 / q}(s, q ; Z),\left|f^{\prime}(b)\right| \leq 1 \leq\left|f^{\prime}(a)\right| ; \\
\left|f^{\prime}(a) f^{\prime}(b)\right|^{1-\frac{s}{2}} M_{1 / 3}^{1 / q}(s, q ; Z)+\left|f^{\prime}(a)\right|^{1-s}\left|f^{\prime}(b)\right| E_{1 / 3}^{1 / q}(s, q ; Z), 1 \leq\left|f^{\prime}(b)\right|,
\end{array}\right.
\end{aligned}
$$

where $Z(u, v), M_{\lambda}(s, q ; Z), E_{\mu}(s, q ; Z)$ are defined in Theorem 3.2.

Theorem 3.3. Let $f: I \subseteq \mathbb{R}_{+} \rightarrow \mathbb{R}_{+}$be differentiable on $I^{\circ}, a, b \in I$ with $a<b$, $0 \leq \lambda, \mu \leq 1$, and $f^{\prime}$ is integrable on $[a, b]$. If $\left|f^{\prime}(x)\right|^{q}$ is s-geometrically convex and monotonically decreasing on $[a, b]$, for $p, q \geq 1$ with $\frac{1}{p}+\frac{1}{q}=1$ and $s \in(0,1]$, then

$$
\begin{gathered}
\left|\frac{\lambda f(a)+\mu f(b)}{2}+\frac{2-\lambda-\mu}{2} f\left(\frac{a+b}{2}\right)-\frac{1}{b-a} \int_{a}^{b} f(x) d x\right| \leq \frac{(b-a)}{4} T^{1 / q}(u) \\
\left\{\begin{array}{cc}
\left|f^{\prime}(a) f^{\prime}(b)\right|^{\frac{s}{2}} F_{\lambda}^{1 / p}+\left|f^{\prime}(b)\right|^{s} F_{\mu}^{1 / p}, & \left|f^{\prime}(a)\right| \leq 1 ; \\
\left|f^{\prime}(a)\right|^{1-\frac{s}{2}}\left|f^{\prime}(b)\right|^{\frac{s}{2}} F_{\lambda}^{1 / p}+\left|f^{\prime}(a)\right|^{1-s}\left|f^{\prime}(b)\right|^{s} F_{\mu}^{1 / p}, & \left|f^{\prime}(b)\right| \leq 1 \leq\left|f^{\prime}(a)\right| ; \\
\left|f^{\prime}(a) f^{\prime}(b)\right|^{1-s / 2} F_{\lambda}^{1 / p}+\left|f^{\prime}(a)\right|^{1-s}\left|f^{\prime}(b)\right| F_{\mu}^{1 / p}, & 1 \leq\left|f^{\prime}(b)\right|,
\end{array}\right.
\end{gathered}
$$

where

$$
T(u)=\left\{\begin{array}{cc}
\frac{u-1}{\ln u}, & u \neq 1 \\
1, & u=1
\end{array}, u=\left|f^{\prime}(a) / f^{\prime}(b)\right|^{\frac{s q}{2}}\right.
$$

and

$$
F_{\lambda}=\frac{\lambda^{p+1}+(1-\lambda)^{p+1}}{p+1}, F_{\mu}=\frac{\mu^{p+1}+(1-\mu)^{p+1}}{p+1} .
$$

Proof. From Lemma 2.1 and since $\left|f^{\prime}(x)\right|^{q}$ is s-geometrically convex and monotonically decreasing on $[a, b]$, by using Hölder's inequality, we get

$$
\begin{aligned}
& \left|\frac{\lambda f(a)+\mu f(b)}{2}+\frac{2-\lambda-\mu}{2} f\left(\frac{a+b}{2}\right)-\frac{1}{b-a} \int_{a}^{b} f(x) d x\right| \\
\leq & \frac{b-a}{4}\left[\left(\int_{0}^{1}|1-\lambda-t|^{p} d t\right)^{\frac{1}{p}}\left(\int_{0}^{1}\left|f^{\prime}\left(a^{\frac{1+t}{2}} b^{\frac{1-t}{2}}\right)\right|^{q} d t\right)^{\frac{1}{q}}\right. \\
& \left.+\left(\int_{0}^{1}|\mu-t|^{p} d t\right)^{\frac{1}{p}}\left(\int_{0}^{1}\left|f^{\prime}\left(a^{\frac{t}{2}} b^{\frac{2-t}{2}}\right)\right|^{q} d t\right)^{\frac{1}{q}}\right] \\
\leq & \frac{b-a}{4}\left[\left(\int_{0}^{1}|1-\lambda-t|^{p} d t\right)^{\frac{1}{p}}\left(\int_{0}^{1}\left(\left|f^{\prime}(a)\right|^{\left(\frac{1+t}{2}\right)^{s}}\left|f^{\prime}(b)\right|^{\left(\frac{1-t}{2}\right)^{s}}\right)^{q} d t\right)^{\frac{1}{q}}\right. \\
& \left.+\left(\int_{0}^{1}|\mu-t|^{p} d t\right)^{\frac{1}{p}}\left(\int_{0}^{1}\left(\left|f^{\prime}(a)\right|^{\left(\frac{t}{2}\right)^{s}}\left|f^{\prime}(b)\right|^{\left(\frac{2-t}{2}\right)^{s}}\right)^{q} d t\right)^{\frac{1}{q}}\right] .
\end{aligned}
$$


It is know that for $0 \leq \lambda, \mu \leq 1$, by using Lemma 2.2 , we have

$$
\int_{0}^{1}|(1-\lambda-t)|^{p} d t=\frac{\lambda^{p+1}+(1-\lambda)^{p+1}}{p+1}=F_{\lambda}
$$

and

$$
\int_{0}^{1}|(\mu-t)|^{p} d t=\frac{\mu^{p+1}+(1-\mu)^{p+1}}{p+1}=F_{\mu} .
$$

Therefore, we have

$$
\begin{aligned}
& \left|\frac{\lambda f(a)+\mu f(b)}{2}+\frac{2-\lambda-\mu}{2} f\left(\frac{a+b}{2}\right)-\frac{1}{b-a} \int_{a}^{b} f(x) d x\right| \\
\leq & \frac{b-a}{4}\left[\left(\frac{\lambda^{p+1}+(1-\lambda)^{p+1}}{p+1}\right)^{\frac{1}{p}}\left(\int_{0}^{1}\left|f^{\prime}(a)\right|^{q\left(\frac{1+t}{2}\right)^{s}}\left|f^{\prime}(b)\right|^{q\left(\frac{1-t}{2}\right)^{s}} d t\right)^{\frac{1}{q}}\right. \\
& \left.+\left(\frac{\mu^{p+1}+(1-\mu)^{p+1}}{p+1}\right)^{\frac{1}{p}}\left(\int_{0}^{1}\left|f^{\prime}(a)\right|^{q\left(\frac{t}{2}\right)^{s}}\left|f^{\prime}(b)\right|^{q\left(\frac{2-t}{2}\right)^{s}} d t\right)^{\frac{1}{q}}\right] .
\end{aligned}
$$

When $\left|f^{\prime}(a)\right| \leq 1$, from (2.3), we get

$$
\begin{aligned}
\int_{0}^{1}\left|f^{\prime}(a)\right|^{q\left(\frac{1+t}{2}\right)^{s}}\left|f^{\prime}(b)\right|^{q\left(\frac{1-t}{2}\right)^{s}} d t & \leq \int_{0}^{1}\left|f^{\prime}(a)\right|^{s q\left(\frac{1+t}{2}\right)}\left|f^{\prime}(b)\right|^{s q\left(\frac{1-t}{2}\right)} d t \\
& =\left|f^{\prime}(a) f^{\prime}(b)\right|^{\frac{s q}{2}} \int_{0}^{1}\left|f^{\prime}(a) / f^{\prime}(b)\right|^{\frac{s q t}{2}} d t \\
& =\left|f^{\prime}(a) f^{\prime}(b)\right|^{\frac{s q}{2}} \frac{Z\left(\frac{s q}{2}, \frac{s q}{2}\right)-1}{\ln Z\left(\frac{s q}{2}, \frac{s q}{2}\right)},
\end{aligned}
$$

and

$$
\begin{aligned}
\int_{0}^{1}\left|f^{\prime}(a)\right|^{q\left(\frac{t}{2}\right)^{s}}\left|f^{\prime}(b)\right|^{q\left(\frac{2-t}{2}\right)^{s}} d t & \leq \int_{0}^{1}\left|f^{\prime}(a)\right|^{\frac{s q t}{2}}\left|f^{\prime}(b)\right|^{s q-\frac{s q t}{2}} d t \\
& =\left|f^{\prime}(b)\right|^{s q} \int_{0}^{1}\left|f^{\prime}(a) / f^{\prime}(b)\right|^{\frac{s q t}{2}} d t \\
& =\left|f^{\prime}(b)\right|^{s q} \frac{Z\left(\frac{s q}{2}, \frac{s q}{2}\right)-1}{\ln Z\left(\frac{s q}{2}, \frac{s q}{2}\right)} .
\end{aligned}
$$

When $\left|f^{\prime}(b)\right| \leq 1 \leq\left|f^{\prime}(a)\right|$, by virtue of (2.3), we get

$$
\begin{aligned}
\int_{0}^{1}\left|f^{\prime}(a)\right|^{q\left(\frac{1+t}{2}\right)^{s}}\left|f^{\prime}(b)\right|^{q\left(\frac{1-t}{2}\right)^{s}} d t & \leq \int_{0}^{1}\left|f^{\prime}(a)\right|^{q[s((1+t) / 2)+1-s]}\left|f^{\prime}(b)\right|^{s q(1-t) / 2} d t \\
& =\left|f^{\prime}(a)\right|^{(1-s / 2) q}\left|f^{\prime}(b)\right|^{s q / 2} \int_{0}^{1}\left|f^{\prime}(a) / f^{\prime}(b)\right|^{s q t / 2} d t \\
& =\left|f^{\prime}(a)\right|^{(1-s / 2) q}\left|f^{\prime}(b)\right|^{s q / 2} \frac{Z\left(\frac{s q}{2}, \frac{s q}{2}\right)-1}{\ln Z\left(\frac{s q}{2}, \frac{s q}{2}\right)},
\end{aligned}
$$


and

$$
\begin{aligned}
\int_{0}^{1}\left|f^{\prime}(a)\right|^{q\left(\frac{t}{2}\right)^{s}}\left|f^{\prime}(b)\right|^{q\left(\frac{2-t}{2}\right)^{s}} d t & \leq \int_{0}^{1}\left|f^{\prime}(a)\right|^{q[s t / 2+1-s]}\left|f^{\prime}(b)\right|^{s q-s q t / 2} d t \\
& =\left|f^{\prime}(a)\right|^{q(1-s)}\left|f^{\prime}(b)\right|^{s q} \int_{0}^{1}\left|f^{\prime}(a) / f^{\prime}(b)\right|^{\frac{s q t}{2}} d t \\
& =\left|f^{\prime}(a)\right|^{q(1-s)}\left|f^{\prime}(b)\right|^{s q} \frac{Z\left(\frac{s q}{2}, \frac{s q}{2}\right)-1}{\ln Z\left(\frac{s q}{2}, \frac{s q}{2}\right)} .
\end{aligned}
$$

When $1 \leq\left|f^{\prime}(b)\right|$, by virtue of $(2.3)$, we get

$$
\begin{aligned}
\int_{0}^{1}\left|f^{\prime}(a)\right|^{q\left(\frac{1+t}{2}\right)^{s}}\left|f^{\prime}(b)\right|^{q\left(\frac{1-t}{2}\right)^{s}} d t & \leq \int_{0}^{1}\left|f^{\prime}(a)\right|^{q[s((1+t) / 2)+1-s]}\left|f^{\prime}(b)\right|^{q[s(1-t) / 2+1-s]} d t \\
& =\left|f^{\prime}(a) f^{\prime}(b)\right|^{(1-s / 2) q} \int_{0}^{1}\left|f^{\prime}(a) / f^{\prime}(b)\right|^{s q t / 2} d t \\
& =\left|f^{\prime}(a) f^{\prime}(b)\right|^{(1-s / 2) q} \frac{Z\left(\frac{s q}{2}, \frac{s q}{2}\right)-1}{\ln Z\left(\frac{s q}{2}, \frac{s q}{2}\right)},
\end{aligned}
$$

and

$$
\begin{aligned}
\int_{0}^{1}\left|f^{\prime}(a)\right|^{q\left(\frac{t}{2}\right)^{s}}\left|f^{\prime}(b)\right|^{q\left(\frac{2-t}{2}\right)^{s}} d t & \leq \int_{0}^{1}\left|f^{\prime}(a)\right|^{q[s t / 2+1-s]}\left|f^{\prime}(b)\right|^{q[1-s t / 2]} d t \\
& =\left|f^{\prime}(a)\right|^{q(1-s)}\left|f^{\prime}(b)\right|^{q} \int_{0}^{1}\left|f^{\prime}(a) / f^{\prime}(b)\right|^{\frac{s q t}{2}} d t \\
& =\left|f^{\prime}(a)\right|^{q(1-s)}\left|f^{\prime}(b)\right|^{q} \frac{Z\left(\frac{s q}{2}, \frac{s q}{2}\right)-1}{\ln Z\left(\frac{s q}{2}, \frac{s q}{2}\right)} .
\end{aligned}
$$

As a result, the proof of Theorem 3.3 is complete.

If taking $\lambda=\mu$ in Theorem 3.3, we derive the following corollary.

Corollary 3.5. Let $f: I \subseteq \mathbb{R}_{+} \rightarrow \mathbb{R}_{+}$be differentiable on $I^{\circ}$, $a, b \in I$, with $a<b$, and $f^{\prime} \in L([a, b])$. If $\left|f^{\prime}(x)\right|$ is s-geometrically convex and monotonically decreasing on $[a, b]$, for $p, q \geq 1$ with $\frac{1}{p}+\frac{1}{q}=1$ and $0<s \leq 1,0 \leq \lambda \leq 1$, then

$$
\begin{aligned}
& \left|\frac{\lambda f(a)+\mu f(b)}{2}+\frac{2-\lambda-\mu}{2} f\left(\frac{a+b}{2}\right)-\frac{1}{b-a} \int_{a}^{b} f(x) d x\right| \\
\leq & \frac{(b-a)}{4} F_{\lambda}^{1 / p} T^{1 / q}(u) \times \\
& \left\{\begin{array}{cc}
\left|f^{\prime}(a) f^{\prime}(b)\right|^{\frac{s}{2}}+\left|f^{\prime}(b)\right|, & \left|f^{\prime}(a)\right| \leq 1 ; \\
\left|f^{\prime}(a)\right|^{1-\frac{s}{2}}\left|f^{\prime}(b)\right|^{\frac{s}{2}}+\left|f^{\prime}(a)\right|^{1-s}\left|f^{\prime}(b)\right|^{s}, & \left|f^{\prime}(b)\right| \leq 1 \leq\left|f^{\prime}(a)\right| ; \\
\left|f^{\prime}(a) f^{\prime}(b)\right|^{1-s / 2}+\left|f^{\prime}(a)\right|^{1-s}\left|f^{\prime}(b)\right|, & 1 \leq\left|f^{\prime}(b)\right|,
\end{array}\right.
\end{aligned}
$$

where $u, T(u), F_{\lambda}$ are defined in Theorem 3.3.

If letting $\lambda=\mu=1 / 2,2 / 3,1 / 3$, respectively, in Theorem 3.3 , we can deduce the inequalities below.

Corollary 3.6. Let $f: I \subseteq \mathbb{R}_{+} \rightarrow \mathbb{R}_{+}$be differentiable on $I^{\circ}, a, b \in I$, with $a<b$, and $f^{\prime} \in L([a, b])$. If $\left|f^{\prime}(x)\right|$ is s-geometrically convex and monotonically 
decreasing on $[a, b]$, for $p, q \geq 1$ with $\frac{1}{p}+\frac{1}{q}=1$ and $0<s \leq 1,0 \leq \lambda \leq 1$, then

$$
\begin{aligned}
& \left|\frac{1}{2}\left[\frac{f(a)+f(b)}{2}+f\left(\frac{a+b}{2}\right)\right]-\frac{1}{b-a} \int_{a}^{b} f(x) d x\right| \\
\leq & \frac{(b-a)}{8(p+1)^{\frac{1}{p}} T^{1 / q}(u) \times} \\
& \left\{\begin{array}{cc}
\left|f^{\prime}(a) f^{\prime}(b)\right|^{\frac{s}{2}}+\left|f^{\prime}(b)\right|, & \left|f^{\prime}(a)\right| \leq 1 ; \\
\left|f^{\prime}(a)\right|^{1-\frac{s}{2}}\left|f^{\prime}(b)\right|^{\frac{s}{2}}+\left|f^{\prime}(a)\right|^{1-s}\left|f^{\prime}(b)\right|^{s}, & \left|f^{\prime}(b)\right| \leq 1 \leq\left|f^{\prime}(a)\right| ; \\
\left|f^{\prime}(a) f^{\prime}(b)\right|^{1-s / 2}+\left|f^{\prime}(a)\right|^{1-s}\left|f^{\prime}(b)\right|, & 1 \leq\left|f^{\prime}(b)\right| ;
\end{array}\right.
\end{aligned}
$$

$$
\begin{aligned}
& \left|\frac{1}{3}\left[f(a)+f(b)+f\left(\frac{a+b}{2}\right)\right]-\frac{1}{b-a} \int_{a}^{b} f(x) d x\right| \\
\leq & \frac{(b-a)}{4}\left(\frac{2^{p+1}+1}{3^{p+1}(p+1)}\right)^{\frac{1}{p}} T^{1 / q}(u) \times \\
& \left\{\begin{array}{cc}
\left|f^{\prime}(a) f^{\prime}(b)\right|^{\frac{s}{2}}+\left|f^{\prime}(b)\right|, & \left|f^{\prime}(a)\right| \leq 1 ; \\
\left|f^{\prime}(a)\right|^{1-\frac{s}{2}}\left|f^{\prime}(b)\right|^{\frac{s}{2}}+\left|f^{\prime}(a)\right|^{1-s}\left|f^{\prime}(b)\right|^{s}, & \left|f^{\prime}(b)\right| \leq 1 \leq\left|f^{\prime}(a)\right| ; \\
\left|f^{\prime}(a) f^{\prime}(b)\right|^{1-s / 2}+\left|f^{\prime}(a)\right|^{1-s}\left|f^{\prime}(b)\right|, & 1 \leq\left|f^{\prime}(b)\right| ;
\end{array}\right.
\end{aligned}
$$

$$
\begin{aligned}
& \left|\frac{1}{6}\left[f(a)+f(b)+4 f\left(\frac{a+b}{2}\right)\right]-\frac{1}{b-a} \int_{a}^{b} f(x) d x\right| \\
\leq & \frac{(b-a)}{4}\left(\frac{2^{p+1}+1}{3^{p+1}(p+1)}\right)^{\frac{1}{p}} T^{1 / q}(u) \times \\
& \left\{\begin{array}{cc}
\left|f^{\prime}(a) f^{\prime}(b)\right|^{\frac{s}{2}}+\left|f^{\prime}(b)\right|, & \left|f^{\prime}(a)\right| \leq 1 ; \\
\left|f^{\prime}(a)\right|^{1-\frac{s}{2}}\left|f^{\prime}(b)\right|^{\frac{s}{2}}+\left|f^{\prime}(a)\right|^{1-s}\left|f^{\prime}(b)\right|^{s}, & \left|f^{\prime}(b)\right| \leq 1 \leq\left|f^{\prime}(a)\right| ; \\
\left|f^{\prime}(a) f^{\prime}(b)\right|^{1-s / 2}+\left|f^{\prime}(a)\right|^{1-s}\left|f^{\prime}(b)\right|, & 1 \leq\left|f^{\prime}(b)\right|,
\end{array}\right.
\end{aligned}
$$

where $u, T(u)$ are defined in Theorem 3.3.

Theorem 3.4. Let $f: I \subseteq \mathbb{R}_{+} \rightarrow \mathbb{R}_{+}$be differentiable on $I^{\circ}, a, b \in I$ with $a<b$, $0 \leq \lambda, \mu \leq 1$, and $f^{\prime}$ is integrable on $[a, b]$. If $\left|f^{\prime}(x)\right|$ is s-geometrically convex and monotonically decreasing on $[a, b]$, for $s \in(0,1]$ and $\omega, \beta>0$ with $\omega+\beta=1$, then

$$
\begin{aligned}
& \left|\frac{\lambda f(a)+\mu f(b)}{2}+\frac{2-\lambda-\mu}{2} f\left(\frac{a+b}{2}\right)-\frac{1}{b-a} \int_{a}^{b} f(x) d x\right| \leq \frac{(b-a)}{4} \times
\end{aligned}
$$

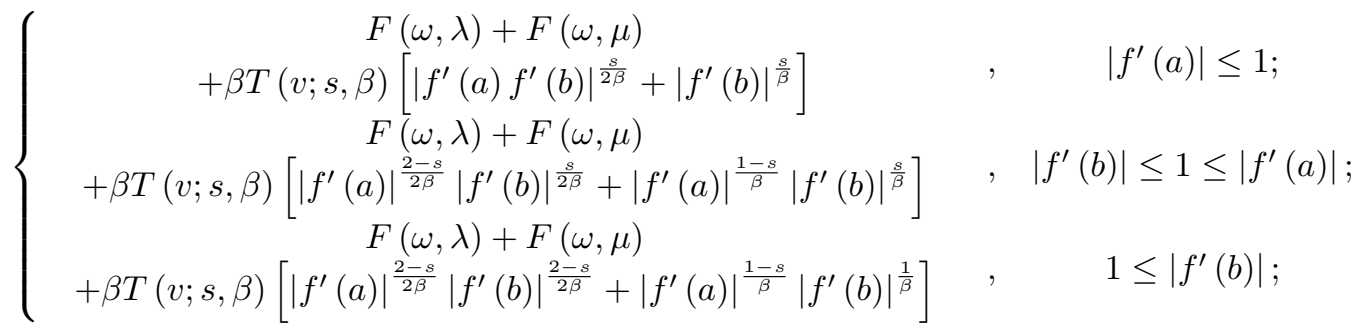


where

$$
T(v ; s, \beta)=\left\{\begin{array}{cc}
\frac{v-1}{\ln v}, & v \neq 1 \\
1, & v=1
\end{array}, v=\left|f^{\prime}(a) / f^{\prime}(b)\right|^{\frac{s}{2 \beta}}\right.
$$

and

$F(\omega, \lambda)=\frac{\omega^{2}}{\omega+1}\left(\lambda^{\frac{1}{\omega}+1}+(1-\lambda)^{\frac{1}{\omega}+1}\right), F(\omega, \mu)=\frac{\omega^{2}}{\omega+1}\left(\mu^{\frac{1}{\omega}+1}+(1-\mu)^{\frac{1}{\omega}+1}\right)$.

Proof. From Lemma 2.1, and since $\left|f^{\prime}(x)\right|$ is s-geometrically convex and monotonically decreasing on $[a, b]$, and by using Cauchy's inequality, we get

$$
\begin{aligned}
& \left|\frac{\lambda f(a)+\mu f(b)}{2}+\frac{2-\lambda-\mu}{2} f\left(\frac{a+b}{2}\right)-\frac{1}{b-a} \int_{a}^{b} f(x) d x\right| \\
\leq & \frac{b-a}{4}\left[\omega \int_{0}^{1}|1-\lambda-t|^{\frac{1}{\omega}} d t+\beta \int_{0}^{1}\left|f^{\prime}\left(t a+(1-t) \frac{a+b}{2}\right)\right|^{\frac{1}{\beta}} d t\right. \\
\leq & \frac{b-a}{4}\left[\omega \int_{0}^{1}|1-\lambda-t|^{\frac{1}{\omega}} d t+\beta \int_{0}^{1}\left|f^{\prime}\left(a^{\frac{1+t}{2}} b^{\frac{1-t}{2}}\right)\right|^{\frac{1}{\beta}} d t\right. \\
& \left.\omega \int_{0}^{1}|\mu-t|^{\frac{1}{\omega}} d t+\beta \int_{0}^{1}\left|f^{\prime}\left(a^{\frac{t}{2}} b^{\frac{2-t}{2}}\right)\right|^{\frac{1}{\beta}} d t\right] \\
\leq & \frac{b-a}{4}\left[\omega \int_{0}^{1}|1-\lambda-t|^{\frac{1}{\omega}} d t+\beta \int_{0}^{1}\left(\left|f^{\prime}(a)\right|^{\left(\frac{1+t}{2}\right)^{s}}\left|f^{\prime}(b)\right|^{\left(\frac{1-t}{2}\right)^{s}}\right)^{\frac{1}{\beta}} d t\right. \\
& +\omega \int_{0}^{1}|\mu-t|^{\frac{1}{\omega}} d t+\beta \int_{0}^{1}\left(\left|f^{\prime}(a)\right|^{\left(\frac{t}{2}\right)^{s}}\left|f^{\prime}(b)\right|^{\left.\left.\left(\frac{2-t}{2}\right)^{s}\right)^{\frac{1}{\beta}} d t\right] .}\right.
\end{aligned}
$$

On the other hand, we have

$$
\omega \int_{0}^{1}|1-\lambda-t|^{\frac{1}{\omega}} d t=\frac{\omega^{2}}{\omega+1}\left(\lambda^{\frac{1}{\omega}+1}+(1-\lambda)^{\frac{1}{\omega}+1}\right)=F(\omega, \lambda)
$$

and

$$
\omega \int_{0}^{1}|\mu-t|^{\frac{1}{\omega}} d t=\frac{\omega^{2}}{\omega+1}\left(\mu^{\frac{1}{\omega}+1}+(1-\mu)^{\frac{1}{\omega}+1}\right)=F(\omega, \mu)
$$

Then, we have

$$
\begin{aligned}
& \left|\frac{\lambda f(a)+\mu f(b)}{2}+\frac{2-\lambda-\mu}{2} f\left(\frac{a+b}{2}\right)-\frac{1}{b-a} \int_{a}^{b} f(x) d x\right| \\
\leq & \frac{b-a}{4}\left[F(\omega, \lambda)+\beta \int_{0}^{1}\left(\left|f^{\prime}(a)\right|^{\left(\frac{1+t}{2}\right)^{s}}\left|f^{\prime}(b)\right|^{\left(\frac{1-t}{2}\right)^{s}}\right)^{\frac{1}{\beta}} d t\right. \\
& \left.+F(\omega, \mu)+\beta \int_{0}^{1}\left(\left|f^{\prime}(a)\right|^{\left(\frac{t}{2}\right)^{s}}\left|f^{\prime}(b)\right|^{\left(\frac{2-t}{2}\right)^{s}}\right)^{\frac{1}{\beta}} d t\right] .
\end{aligned}
$$


When $\left|f^{\prime}(a)\right| \leq 1$, from (2.3), we get

$$
\begin{aligned}
\beta \int_{0}^{1}\left(\left|f^{\prime}(a)\right|^{\left(\frac{1+t}{2}\right)^{s}}\left|f^{\prime}(b)\right|^{\left(\frac{1-t}{2}\right)^{s}}\right)^{\frac{1}{\beta}} d t & \leq \beta \int_{0}^{1}\left|f^{\prime}(a)\right|^{\frac{s+s t}{2 \beta}}\left|f^{\prime}(b)\right|^{\frac{s-s t}{2 \beta}} d t \\
& =\beta\left|f^{\prime}(a) f^{\prime}(b)\right|^{\frac{s}{2 \beta}} \int_{0}^{1}\left|f^{\prime}(a) / f^{\prime}(b)\right|^{\frac{s t}{2 \beta}} d t \\
& =\beta\left|f^{\prime}(a) f^{\prime}(b)\right|^{\frac{s}{2 \beta}} T(v ; s, \beta),
\end{aligned}
$$

and

$$
\begin{aligned}
\beta \int_{0}^{1}\left(\left|f^{\prime}(a)\right|^{\left(\frac{t}{2}\right)^{s}}\left|f^{\prime}(b)\right|^{\left(\frac{2-t}{2}\right)^{s}}\right)^{\frac{1}{\beta}} d t & \leq \beta \int_{0}^{1}\left|f^{\prime}(a)\right|^{\frac{s t}{2 \beta}}\left|f^{\prime}(b)\right|^{\frac{2 s-s t}{2 \beta}} d t \\
& =\beta\left|f^{\prime}(b)\right|^{\frac{s}{\beta}} \int_{0}^{1}\left|f^{\prime}(a) / f^{\prime}(b)\right|^{\frac{s t}{2 \beta}} d t \\
& =\beta\left|f^{\prime}(b)\right|^{\frac{s}{\beta}} T(v ; s, \beta) .
\end{aligned}
$$

When $\left|f^{\prime}(b)\right| \leq 1 \leq\left|f^{\prime}(a)\right|$, by virtue of (2.3), we get

$$
\begin{aligned}
\beta \int_{0}^{1}\left(\left|f^{\prime}(a)\right|^{\left(\frac{1+t}{2}\right)^{s}}\left|f^{\prime}(b)\right|^{\left(\frac{1-t}{2}\right)^{s}}\right)^{\frac{1}{\beta}} d t & \leq \beta \int_{0}^{1}\left|f^{\prime}(a)\right|^{\frac{s t+2-s}{2 \beta}}\left|f^{\prime}(b)\right|^{\frac{s-s t}{2 \beta}} d t \\
& =\beta\left|f^{\prime}(a)\right|^{\frac{2-s}{2 \beta}}\left|f^{\prime}(b)\right|^{\frac{s}{2 \beta}} \int_{0}^{1}\left|f^{\prime}(a) / f^{\prime}(b)\right|^{\frac{s t}{2 \beta}} d t \\
& =\beta\left|f^{\prime}(a)\right|^{\frac{2-s}{2 \beta}}\left|f^{\prime}(b)\right|^{\frac{s}{2 \beta}} T(v ; s, \beta),
\end{aligned}
$$

and

$$
\begin{aligned}
\beta \int_{0}^{1}\left(\left|f^{\prime}(a)\right|^{\left(\frac{t}{2}\right)^{s}}\left|f^{\prime}(b)\right|^{\left(\frac{2-t}{2}\right)^{s}}\right)^{\frac{1}{\beta}} d t & \leq \beta \int_{0}^{1}\left|f^{\prime}(a)\right|^{\frac{s t+2-2 s}{2 \beta}}\left|f^{\prime}(b)\right|^{\frac{2 s-s t}{2 \beta}} d t \\
& =\beta\left|f^{\prime}(a)\right|^{\frac{1-s}{\beta}}\left|f^{\prime}(b)\right|^{\frac{s}{\beta}} \int_{0}^{1}\left|f^{\prime}(a) / f^{\prime}(b)\right|^{\frac{s t}{2 \beta}} d t \\
& =\beta\left|f^{\prime}(a)\right|^{\frac{1-s}{\beta}}\left|f^{\prime}(b)\right|^{\frac{s}{\beta}} T(v ; s, \beta) .
\end{aligned}
$$

When $1 \leq\left|f^{\prime}(b)\right|$, by using (2.3), we get

$$
\begin{aligned}
\beta \int_{0}^{1}\left(\left|f^{\prime}(a)\right|^{\left(\frac{1+t}{2}\right)^{s}}\left|f^{\prime}(b)\right|^{\left(\frac{1-t}{2}\right)^{s}}\right)^{\frac{1}{\beta}} d t & \leq \beta \int_{0}^{1}\left|f^{\prime}(a)\right|^{\frac{s t+2-s}{2 \beta}}\left|f^{\prime}(b)\right|^{\frac{2-s-s t}{2 \beta}} d t \\
& =\beta\left|f^{\prime}(a)\right|^{\frac{2-s}{2 \beta}}\left|f^{\prime}(b)\right|^{\frac{2-s}{2 \beta}} \int_{0}^{1}\left|f^{\prime}(a) / f^{\prime}(b)\right|^{\frac{s t}{2 \beta}} d t \\
& =\beta\left|f^{\prime}(a)\right|^{\frac{2-s}{2 \beta}}\left|f^{\prime}(b)\right|^{\frac{2-s}{2 \beta}} T(v ; s, \beta),
\end{aligned}
$$

and

$$
\begin{aligned}
\beta \int_{0}^{1}\left(\left|f^{\prime}(a)\right|^{\left(\frac{t}{2}\right)^{s}}\left|f^{\prime}(b)\right|^{\left(\frac{2-t}{2}\right)^{s}}\right)^{\frac{1}{\beta}} d t & \leq \beta \int_{0}^{1}\left|f^{\prime}(a)\right|^{\frac{s t+2-2 s}{2 \beta}}\left|f^{\prime}(b)\right|^{\frac{2-s t}{2 \beta}} d t \\
& =\beta\left|f^{\prime}(a)\right|^{\frac{1-s}{\beta}}\left|f^{\prime}(b)\right|^{\frac{1}{\beta}} \int_{0}^{1}\left|f^{\prime}(a) / f^{\prime}(b)\right|^{\frac{s t}{2 \beta}} d t \\
& =\beta\left|f^{\prime}(a)\right|^{\frac{1-s}{\beta}}\left|f^{\prime}(b)\right|^{\frac{1}{\beta}} T(v ; s, \beta) .
\end{aligned}
$$

The proof of Theorem 3.4 is complete.

If taking $\lambda=\mu$ in Theorem 3.4, we derive the following corollary. 
Corollary 3.7. Let $f: I \subseteq \mathbb{R}_{+} \rightarrow \mathbb{R}_{+}$be differentiable on $I^{\circ}, a, b \in I$ with $a<b$, $0 \leq \lambda \leq 1$, and $f^{\prime}$ is integrable on $[a, b]$. If $\left|f^{\prime}(x)\right|^{q}$ is s-geometrically convex and monotonically decreasing on $[a, b]$, for $s \in(0,1]$ and $\omega, \beta>0$ with $\omega+\beta=1$, then

$$
\begin{aligned}
& \left|\frac{\lambda f(a)+\mu f(b)}{2}+\frac{2-\lambda-\mu}{2} f\left(\frac{a+b}{2}\right)-\frac{1}{b-a} \int_{a}^{b} f(x) d x\right| \leq \frac{b-a}{4} \\
& \times\left\{\begin{array}{c}
2 F(\omega, \lambda)+\beta T(v ; s, \beta)\left[\left|f^{\prime}(a) f^{\prime}(b)\right|^{\frac{s}{2 \beta}}+\left|f^{\prime}(b)\right|^{\frac{s}{\beta}}\right], \quad\left|f^{\prime}(a)\right| \leq 1 ; \\
2 F(\omega, \lambda)+\beta T(v ; s, \beta)\left[\left|f^{\prime}(a)\right|^{\frac{2-s}{2 \beta}}\left|f^{\prime}(b)\right|^{\frac{s}{2 \beta}}+\left|f^{\prime}(a)\right|^{\frac{1-s}{\beta}}\left|f^{\prime}(b)\right|^{\frac{s}{\beta}}\right], \quad\left|f^{\prime}(b)\right| \leq 1 \leq\left|f^{\prime}(a)\right| ; \\
2 F(\omega, \lambda)+\beta T(v ; s, \beta)\left[\left|f^{\prime}(a)\right|^{\frac{2-s}{2 \beta}}\left|f^{\prime}(b)\right|^{\frac{2-s}{2 \beta}}+\left|f^{\prime}(a)\right|^{\frac{1-s}{\beta}}\left|f^{\prime}(b)\right|^{\frac{1}{\beta}}\right], \quad 1 \leq\left|f^{\prime}(b)\right|,
\end{array}\right.
\end{aligned}
$$

where $T(v ; s, \beta), F(\omega, \lambda)$ are defined in Theorem 3.4.

If letting $\lambda=\mu=1 / 2,2 / 3,1 / 3$, respectively, in Theorem 3.4, we can deduce the inequalities below.

Corollary 3.8. Let $f: I \subseteq \mathbb{R}_{+} \rightarrow \mathbb{R}_{+}$be differentiable on $I^{\circ}, a, b \in I$ with $a<b$, $0 \leq \lambda, \mu \leq 1$, and $f^{\prime}$ is integrable on $[a, b]$. If $\left|f^{\prime}(x)\right|^{q}$ is s-geometrically convex and monotonically decreasing on $[a, b]$, for $s \in(0,1]$ and $\omega, \beta>0$ with $\omega+\beta=1$, then

$$
\begin{aligned}
& \left|\frac{1}{2}\left[\frac{f(a)+f(b)}{2}+f\left(\frac{a+b}{2}\right)\right]-\frac{1}{b-a} \int_{a}^{b} f(x) d x\right| \leq \frac{b-a}{4} \\
& \times\left\{\begin{array}{c}
\frac{\omega^{2}}{2^{1 / \omega-1}(\omega+1)}+\beta T(v ; s, \beta)\left[\left|f^{\prime}(a) f^{\prime}(b)\right|^{\frac{s}{2 \beta}}+\left|f^{\prime}(b)\right|^{\frac{s}{\beta}}\right], \quad\left|f^{\prime}(a)\right| \leq 1 ; \\
\frac{\omega^{2}}{2^{1 / \omega-1}(\omega+1)}+\beta T(v ; s, \beta)\left[\left|f^{\prime}(a)\right|^{\frac{2-s}{2 \beta}}\left|f^{\prime}(b)\right|^{\frac{s}{2 \beta}}+\left|f^{\prime}(a)\right|^{\frac{1-s}{\beta}}\left|f^{\prime}(b)\right|^{\frac{s}{\beta}}\right],\left|f^{\prime}(b)\right| \leq 1 \leq\left|f^{\prime}(a)\right| ; \\
\frac{\omega^{2}}{2^{1 / \omega-1}(\omega+1)}+\beta T(v ; s, \beta)\left[\left|f^{\prime}(a)\right|^{\frac{2-s}{2 \beta}}\left|f^{\prime}(b)\right|^{\frac{2-s}{2 \beta}}+\left|f^{\prime}(a)\right|^{\frac{1-s}{\beta}}\left|f^{\prime}(b)\right|^{\frac{1}{\beta}}\right], \quad 1 \leq\left|f^{\prime}(b)\right| ;
\end{array}\right.
\end{aligned}
$$

$$
\begin{aligned}
& \left|\frac{1}{3}\left[f(a)+f(b)+f\left(\frac{a+b}{2}\right)\right]-\frac{1}{b-a} \int_{a}^{b} f(x) d x\right| \leq \frac{b-a}{4} \\
& \times\left\{\begin{array}{c}
\frac{2 \omega^{2}\left(2^{\frac{1}{\omega}+1}+1\right)}{3^{1 / \omega+1}(\omega+1)}+\beta T(v ; s, \beta)\left[\left|f^{\prime}(a) f^{\prime}(b)\right|^{\frac{s}{2 \beta}}+\left|f^{\prime}(b)\right|^{\frac{s}{\beta}}\right], \quad\left|f^{\prime}(a)\right| \leq 1 ; \\
\frac{2 \omega^{2}\left(2^{\frac{1}{\omega}+1}+1\right)}{3^{1 / \omega+1}(\omega+1)}+\beta T(v ; s, \beta)\left[\left|f^{\prime}(a)\right|^{\frac{2-s}{2 \beta}}\left|f^{\prime}(b)\right|^{\frac{s}{2 \beta}}+\left|f^{\prime}(a)\right|^{\frac{1-s}{\beta}}\left|f^{\prime}(b)\right|^{\frac{s}{\beta}}\right],\left|f^{\prime}(b)\right| \leq 1 \leq\left|f^{\prime}(a)\right| ; \\
\frac{2 \omega^{2}\left(2^{\frac{1}{\omega}+1}+1\right)}{3^{1 / \omega+1}(\omega+1)}+\beta T(v ; s, \beta)\left[\left|f^{\prime}(a)\right|^{\frac{2-s}{2 \beta}}\left|f^{\prime}(b)\right|^{\frac{2-s}{2 \beta}}+\left|f^{\prime}(a)\right|^{\frac{1-s}{\beta}}\left|f^{\prime}(b)\right|^{\frac{1}{\beta}}\right], \quad 1 \leq\left|f^{\prime}(b)\right| ;
\end{array}\right.
\end{aligned}
$$

$$
\begin{aligned}
& \left|\frac{1}{6}\left[f(a)+f(b)+4 f\left(\frac{a+b}{2}\right)\right]-\frac{1}{b-a} \int_{a}^{b} f(x) d x\right| \leq \frac{b-a}{4} \\
& \times\left\{\begin{array}{c}
\frac{2 \omega^{2}\left(2^{\frac{1}{\omega}+1}+1\right)}{3^{1 / \omega+1}(\omega+1)}+\beta T(v ; s, \beta)\left[\left|f^{\prime}(a) f^{\prime}(b)\right|^{\frac{s}{2 \beta}}+\left|f^{\prime}(b)\right|^{\frac{s}{\beta}}\right], \quad\left|f^{\prime}(a)\right| \leq 1 ; \\
\frac{2 \omega^{2}\left(2^{\frac{1}{\omega}+1}+1\right)}{3^{1 / \omega+1}(\omega+1)}+\beta T(v ; s, \beta)\left[\left|f^{\prime}(a)\right|^{\frac{2-s}{2 \beta}}\left|f^{\prime}(b)\right|^{\frac{s}{2 \beta}}+\left|f^{\prime}(a)\right|^{\frac{1-s}{\beta}}\left|f^{\prime}(b)\right|^{\frac{s}{\beta}}\right],\left|f^{\prime}(b)\right| \leq 1 \leq\left|f^{\prime}(a)\right| ; \\
\frac{2 \omega^{2}\left(2^{\frac{1}{\omega}+1}+1\right)}{3^{1 / \omega+1}(\omega+1)}+\beta T(v ; s, \beta)\left[\left|f^{\prime}(a)\right|^{\frac{2-s}{2 \beta}}\left|f^{\prime}(b)\right|^{\frac{2-s}{2 \beta}}+\left|f^{\prime}(a)\right|^{\frac{1-s}{\beta}}\left|f^{\prime}(b)\right|^{\frac{1}{\beta}}\right], \quad 1 \leq\left|f^{\prime}(b)\right| ;
\end{array}\right.
\end{aligned}
$$


where $T(v ; s, \beta)$ as in the Theorem 3.4.

If letting $\lambda=\mu=1 / 2,2 / 3,1 / 3$, and $\omega, \beta=1 / 2$ respectively, in Theorem 3.4 , we can deduce the inequalities below.

Corollary 3.9. Let $f: I \subseteq \mathbb{R}_{+} \rightarrow \mathbb{R}_{+}$be differentiable on $I^{\circ}, a, b \in I$ with $a<b$, and $f^{\prime}$ is integrable on $[a, b]$. If $\left|f^{\prime}(x)\right|^{q}$ is s-geometrically convex and monotonically decreasing on $[a, b]$, for $s \in(0,1]$, then

$$
\begin{aligned}
& \left|\frac{1}{2}\left[\frac{f(a)+f(b)}{2}+f\left(\frac{a+b}{2}\right)\right]-\frac{1}{b-a} \int_{a}^{b} f(x) d x\right| \leq \frac{b-a}{8} \\
& \times\left\{\begin{array}{c}
\frac{1}{6}+T\left(v ; s, \frac{1}{2}\right)\left[\left|f^{\prime}(a) f^{\prime}(b)\right|^{s}+\left|f^{\prime}(b)\right|^{2 s}\right], \quad\left|f^{\prime}(a)\right| \leq 1 ; \\
\frac{1}{6}+T\left(v ; s, \frac{1}{2}\right)\left[\left|f^{\prime}(a)\right|^{2-s}\left|f^{\prime}(b)\right|^{s}+\left|f^{\prime}(a)\right|^{2-2 s}\left|f^{\prime}(b)\right|^{2 s}\right], \quad\left|f^{\prime}(b)\right| \leq 1 \leq\left|f^{\prime}(a)\right| ; \\
\frac{1}{6}+T\left(v ; s, \frac{1}{2}\right)\left[\left|f^{\prime}(a) f^{\prime}(b)\right|^{2-s}+\left|f^{\prime}(a)\right|^{2-2 s}\left|f^{\prime}(b)\right|^{2}\right], \quad 1 \leq\left|f^{\prime}(b)\right| ;
\end{array}\right.
\end{aligned}
$$

$$
\begin{aligned}
& \left|\frac{1}{3}\left[f(a)+f(b)+f\left(\frac{a+b}{2}\right)\right]-\frac{1}{b-a} \int_{a}^{b} f(x) d x\right| \leq \frac{b-a}{8} \\
& \times\left\{\begin{array}{c}
\frac{2}{9}+T\left(v ; s, \frac{1}{2}\right)\left[\left|f^{\prime}(a) f^{\prime}(b)\right|^{s}+\left|f^{\prime}(b)\right|^{2 s}\right], \quad\left|f^{\prime}(a)\right| \leq 1 ; \\
\frac{2}{9}+T\left(v ; s, \frac{1}{2}\right)\left[\left|f^{\prime}(a)\right|^{2-s}\left|f^{\prime}(b)\right|^{s}+\left|f^{\prime}(a)\right|^{2-2 s}\left|f^{\prime}(b)\right|^{2 s}\right], \quad\left|f^{\prime}(b)\right| \leq 1 \leq\left|f^{\prime}(a)\right| ; \\
\frac{2}{9}+T\left(v ; s, \frac{1}{2}\right)\left[\left|f^{\prime}(a) f^{\prime}(b)\right|^{2-s}+\left|f^{\prime}(a)\right|^{2-2 s}\left|f^{\prime}(b)\right|^{2}\right], \quad 1 \leq\left|f^{\prime}(b)\right| ;
\end{array}\right.
\end{aligned}
$$

$$
\begin{aligned}
& \left|\frac{1}{6}\left[f(a)+f(b)+4 f\left(\frac{a+b}{2}\right)\right]-\frac{1}{b-a} \int_{a}^{b} f(x) d x\right| \leq \frac{b-a}{8} \\
& \times\left\{\begin{array}{c}
\frac{2}{9}+T\left(v ; s, \frac{1}{2}\right)\left[\left|f^{\prime}(a) f^{\prime}(b)\right|^{s}+\left|f^{\prime}(b)\right|^{2 s}\right], \quad\left|f^{\prime}(a)\right| \leq 1 ; \\
\frac{2}{9}+T\left(v ; s, \frac{1}{2}\right)\left[\left|f^{\prime}(a)\right|^{2-s}\left|f^{\prime}(b)\right|^{s}+\left|f^{\prime}(a)\right|^{2-2 s}\left|f^{\prime}(b)\right|^{2 s}\right], \quad\left|f^{\prime}(b)\right| \leq 1 \leq\left|f^{\prime}(a)\right| ; \\
\frac{2}{9}+T\left(v ; s, \frac{1}{2}\right)\left[\left|f^{\prime}(a) f^{\prime}(b)\right|^{2-s}+\left|f^{\prime}(a)\right|^{2-2 s}\left|f^{\prime}(b)\right|^{2}\right], \quad 1 \leq\left|f^{\prime}(b)\right| ;
\end{array}\right.
\end{aligned}
$$

where $T(v ; s, \beta)$ as in the Theorem 3.4.

\section{REFERENCES}

[1] Alomari, M., Darus, M., Dragomir, S. S., New inequalities of Simpson's type for $s$ convex functions with applications. RGMIA Res. Rep. Coll. 12 (4) (2009) Article 9. Online http://ajmaa.org/RGMIA/v12n4.php.

[2] Alomari, M., Darus, M., Kırmacı, U. S., Refinements of Hadamard-type inequalities for quasiconvex functions with applications to trapezoidal formula and to special means, Comp. and Math. with Appl. Vol.59 (2010), 225-232.

[3] Bai, R.-F., Qi, F., Xi, B.-Y., Hermite-Hadamard type inequalities for the $m$ - and $(\alpha, m)$ logarithmically convex functions. Filomat, 27 (2013), 1-7.

[4] Dragomir, S. S., Agarwal, R. P., Two inequalities for differentiable mappings and applications to special means of real numbers and to trapezoidal formula, Appl. Math. Lett. 11 (1998) no. 5, 91-95.

[5] Dragomir, S. S., Agarwal, R. P., Cerone, P., On Simpson's inequality and applications. J. of Ineq. and Appl., 5 (2000), 533-579. 
[6] Dragomir, S. S., Pearce, C. E. M., Selected topics on Hermite-Hadamard inequalities and applications, RGMIA monographs, Victoria University, 2000. [Online:http://www.staff.vu.edu.au/RGMIA/monographs/hermite-hadamard.html].

[7] Hadamard, J., Étude sur les propriétés des fonctions entières et en particulier d'une fonction considerée par Riemann. J. Math Pures Appl., 58, (1893) 171-215.

[8] Hudzik, H., Maligranda, L., Some remarks on s-convex functions. Aequationes Math., Vol. 48 (1994), 100-111.

[9] Mitrinović, D. S., Pečarić, J., Fink,A. M., Classical and new inequalities in analysis. KluwerAcademic, Dordrecht, 1993.

[10] Pečarić, J. E., Proschan, F. Tong, Y. L., Convex Functions, Partial Orderings, and Statistical Applications. Academic Press Inc., 1992.

[11] Sarikaya, M. Z., Set, E., Özdemir, M.E., On new inequalities of Simpson's type for convex functions. RGMIA Res. Rep. Coll. 13 (2) (2010) Article2.

[12] Sarikaya, M. Z., Set, E., Özdemir, M.E., On new inequalities of Simpson's type for $s$-convex functions. Comp. and Math. with Appl. 60 (2010) 2191-2199.

[13] Tunç, M., On some new inequalities for convex functions. Turk. J. Math. 36 (2012), 245-251.

[14] Xi, B.-Y., Qi, F., Some Integral Inequalities of Hermite-Hadamard Type for Convex Functions with Applications to Means. Journal of Function Spaces and Appl., Volume 2012, Article ID 980438, 14 p., doi:10.1155/2012/980438.

[15] Zhang, T.-Y., Ji, A.-P., Qi, F., On integral inequalities of Hermite-Hadamard type for $s$ geometrically convex function. Abstract and Applied Analysis, doi:10.1155/2012/560586.

[16] Zhang, T.-Y., Tunç, M., Ji, A.-P., Xi, B.-Y., Corrections to the paper "On integral inequalities of Hermite-Hadamard type for $s$-geometrically convex function". Abstract and Applied Analysis, (2014), Article ID 294739, http://dx.doi.org/10.1155/2014/294739 .

*Department of Mathematics, Faculty of Science and Arts, Mustafa Kemal UniverSITY, 31000, HATAY, TURKEY

E-mail address: mevluttttunc@gmail.com

**Department of Mathematics, Faculty of Science and Arts, AĞri İbrahim Çeçen University, 04000, AĞRI, TURKEY

E-mail address: yuksel.ebru90@hotmail.com 